\title{
Modélisation du bruit des jets turbulents libres et subsoniques à température ambiante
}

\author{
W. Béchara $\left({ }^{1}, *\right)$, P. Lafon $\left({ }^{1}\right)$ et S. M. Candel $\left({ }^{2}\right)$ \\ ( ${ }^{1}$ ) Dépt. Acoustique et Mécanique Vibratoire (AMV), Direction des Etudes et Recherches (DER) \\ d'Electricité de France (EDF), 1 av. du Général-de-Gaulle, 92141 Clamart Cedex, France \\ ( $\left.{ }^{2}\right)$ Ecole Centrale Paris (ECP), 92295 Châtenay, Malabry Cedex, France \\ (Reçu le 14 avril 1992, révisé le 16 décembre 1992, accepté le 18 décembre 1992)
}

\begin{abstract}
Résumé. - A partir du modèle théorique proposé initialement par Goldstein pour un jet libre turbulent circulaire, on établit des expressions analytiques (modèle $G_{\mathrm{a}}$ ) permettant le calcul du bruit émis à partir des grandeurs statistiques locales du jet turbulent. Ces grandeurs sont estimées par résolution des équations de Navier-Stokes moyennées à l'aide d'un code numérique de turbulence utilisant un modèle de fermeture de type $K-\varepsilon$. La comparaison entre les résultats numériques obtenus et les données expérimentales, pour un jet simple et deux jets coaxiaux, montre que ce modèle estime correctement l'évolution des grandeurs acoustiques étudiées. Les calculs indiquent que le modèle $G_{\mathrm{a}}$ développé pour une turbulence axisymétrique convectée par un écoulement moyen est mieux adapté que celui de Ribner associé à une turbulence isotrope. De plus, une comparaison entre différents modèles basés sur la théorie de Lighthill indique que le modèle $G_{\mathrm{a}}$ donne la meilleure directivité de l'intensité acoustique aux vitesses d'éjection élevées.
\end{abstract}

\begin{abstract}
Based on the theoretical model of Goldstein for a round free jet, we establish analytical expressions ( $G_{\mathrm{a}}$ model) for the noise radiation from a turbulent jet, depending on the local statistical properties of the flow. These characteristics are calculated by solving the Reynolds average Navier-Stokes equations with a numerical code based on a $K-\varepsilon$ turbulence closure model. A comparison between the numerical results and the experimental data for a simple jet and two coaxial jets shows that this model correctly predicts the evolution of the acoustic radiation. The $G_{\mathrm{a}}$ model developed in the case of axisymmetric turbulence superimposed over a mean flow is found to be more suitable than the Ribner model associated to an isotropic turbulence. A comparison between differents models issued from Lighthill theory, shows that the $G_{\mathrm{a}}$ model yields the best directivity of the acoustic intensity at high jet exit velocities.
\end{abstract}

\section{Introduction.}

Notre compréhension des phénomènes de rayonnement du bruit par des jets turbulents à grand nombre de Reynolds est basée principalement sur l'analogie acoustique introduite par Lighthill

(*) Adresse actuelle : Centre de Recherche Claude Delorme de l'Air Liquide, B.P. 126, 78350 Les Loges en Josas, France. 
$[14,15]$. Pour utiliser cette théorie afin d'estimer l'intensité acoustique à partir des grandeurs statistiques de la turbulence des écoulements cisaillés, il faut mesurer ou déduire analytiquement le tenseur de corrélation en 2 points et 2 temps des dérivées secondes temporelles des contraintes de Reynolds instantanées et intégrer le résultat sur toute la région turbulente. Une évaluation de ce type par une méthode analytique ou exacte utilisant des données expérimentales tridimensionnelles est irréalisable actuellement. En fait, le seul cas où l'émission acoustique a été calculée analytiquement à partir des grandeurs turbulentes est celui de la décroissance d'une turbulence homogène et isotrope (Proudman [19]) mais les résultats obtenus dans ce cas ne sont pas directement applicables au bruit de jets. Des essais d'extension de l'approche de Proudman à l'analyse des jets turbulents ont été réalisés par Lilley [16], Ribner [20-23], Jones [10], Krishnappa [12], Pao [18] et Goldstein et al. [7]. Toutes ces contributions proposent différentes modélisations des sources acoustiques et de l'émission acoustique totale en fonction des grandeurs locales de l'écoulement moyen et de la turbulence, telles que la vitesse axiale de l'écoulement moyen $U_{1}$, la vitesse moyenne de convection $U_{1 \text { c }}$, l'énergie cinétique turbulente $K$, l'échelle intégrale longitudinale de turbulence $L_{1}$ et la pulsation caractéristique de la turbulence $\omega_{\mathrm{f}}$.

Dans ce travail, nous nous proposons de valider une telle approche. On part ici de modèles proposés par Ribner [22] et Goldstein et al. [7]. Ces modèles présentent l'avantage d'être établis avec un nombre d'approximations relativement limité. On va d'abord dégager les expressions qui permettent le calcul du bruit rayonné à partir des grandeurs locales $U_{1}, U_{1}$ c $L_{1}, K$ et $\omega_{\mathrm{f}}$. On montrera ensuite comment ces grandeurs peuvent être estimées. Ainsi par exemple, la vitesse axiale $U_{1}$ et l'énergie $K$ sont directement déterminées par une résolution numérique des équations dynamiques moyennées auxquelles on a ajouté un modèle de turbulence à 2 équations de transport de type $K-\varepsilon$. Le couplage entre un modèle de bruit aérodynamique et un code de modélisation de l'écoulement turbulent ne semble pas avoir été réalisé dans le passé d'une façon aussi précise. Pour réaliser cette opération, il faut donner des règles de calcul des autres grandeurs $U_{1 \mathrm{c}}, L_{1}$ et $\omega_{\mathrm{f}}$ qui ne sont pas directement accessibles par le calcul aérodynamique. On montre par la suite comment on peut obtenir ces grandeurs à partir de $U_{1}, K$ et $\varepsilon$.

Le plan de cet article est le suivant. Dans la section 2, on présente d'abord la démarche théorique suivie pour caractériser les sources acoustiques, puis on développe les deux modèles de bruit à partir de ceux présentés initialement par Ribner [22] et Goldstein et al. [7]. Par rapport à ces travaux, notre démarche a été de remplacer les estimations empiriques souvent adoptées pour calculer les paramètres des modèles par des estimations basées sur des résultats de simulation numérique. Il s'agit là d'une amélioration originale des travaux précédents. A la section 3 , on expose les règles de calcul des grandeurs aérodynamiques locales. Des calculs numériques des grandeurs acoustiques sont ensuite effectués à partir des 2 modèles et les résultats sont comparés aux données expérimentales de Lush [17] (Sect. 4) dans le cas d'un jet turbulent unique. Une application intéressante de notre version du modèle de Goldstein (désigné par la suite par $G_{\mathrm{a}}$ ) est réalisée dans la section 5. Cette application consiste à étudier la diminution de l'émission acoustique de deux jets turbulents coaxiaux en faisant varier la vitesse de sortie du jet secondaire. Notre objectif est d'une part, de retrouver par ce modèle la vitesse du jet secondaire observée expérimentalement [Juvé et al., 11] correspondant à l'émission acoustique minimale, et d'autre part de prévoir la diminution de puissance acoustique mesurée à cette vitesse.

\section{Application de la théorie de Lighthill au bruit des jets.}

On donne ici quelques éléments de base de la théorie du bruit aérodynamique. Les modèles proposés par Ribner et Goldstein sont ensuite examinés. Les expressions qui permettent le 
calcul du champ rayonné à partir des caractéristiques turbulentes locales sont rassemblées et mises sous une forme directement utilisable dans une simulation numérique.

2.1 DÉTERMINATION DE LA FONCTION D'AUTO-CORRÉlATION. - Le bruit rayonné par un volume fini de fluide turbulent $(V)$ situé dans un fluide infini au repos peut être décrit à l'aide de la théorie de Lighthill $[14,15]$. Selon cette théorie, les fluctuations acoustiques de densité en champ lointain dues à la turbulence contenue dans le volume $(V)$ sont déterminées par la relation suivante :

$$
\rho(\mathbf{x}, t)-\rho_{0}=\frac{x_{i} x_{j}}{4 \pi c_{0}^{4} x^{3}} \int_{V} \frac{\partial^{2}}{\partial t^{2}} T_{i j}\left(\mathbf{y}, t-|\mathbf{x}-\mathbf{y}| / c_{0}\right) \mathrm{d} \mathbf{y}
$$

où $\rho_{0}$ et $c_{0}$ sont la masse volumique et la vitesse du son du milieu fluide au repos, $\mathbf{x}$ est le point d'observation en champ lointain, y un point courant du volume source $(V)$ et $t$ est le temps. Les composantes $T_{\imath \jmath}$ du tenseur instantané de Lighthill comprennent un premier terme de génération du bruit lié à l'intensité de la turbulence $\rho u_{t} u_{j}$, un second terme correspondant aux fluctuations d'entropie dans le milieu $\left[\left(p-p_{0}\right)-c_{0}^{2}\left(\rho-\rho_{0}\right)\right] \delta_{\imath j}$ et un troisième terme associé à la dissipation par les effets visqueux $\tau_{i j}$. Pour des nombres de Reynolds grands et des nombres de Mach faibles, en l'absence des sources de chaleur et en négligeant la chaleur dégagée par le travail des forces visqueuses, le tenseur de Lighthill est identifié à $\rho_{0} u_{i} u_{j}$ ( $u_{t}$ étant la composante de la vitesse instantanée selon l'axe $i$ ).

En considérant le cas des jets turbulents stationnaires, nous pouvons définir la fonction d'auto-corrélation $C_{\rho \rho}(\mathbf{x}, \tau)$ de la densité acoustique par :

$$
C_{\rho \rho}(\mathbf{x}, \tau)=\frac{\overline{\left[\rho(\mathbf{x}, t+\tau)-\rho_{0}\right]\left[\rho(\mathbf{x}, t)-\rho_{0}\right]}}{\rho_{0} c_{0}^{-3}}
$$

En vertu de (1), $C_{\rho \rho}(\mathbf{x}, \tau)$ sera liée au terme source par :

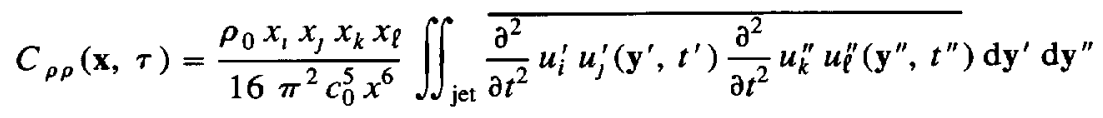

où $t^{\prime}=t-\left|\mathbf{x}-\mathbf{y}^{\prime}\right| / c_{0} ; t^{\prime \prime}=t+\tau-\left|\mathbf{x}-\mathbf{y}^{\prime \prime}\right| / c_{0}, \mathbf{y}^{\prime}$ et $\mathbf{y}^{\prime \prime}$ représentent deux points courants du volume turbulent $(V)$. Dans le cas envisagé ici, $(V)$ s'identifie au volume occupé par le jet turbulent. Goldstein [6] montre que la fonction de corrélation $C_{p \rho}(x, \tau)$ peut se mettre sous la forme :

$$
C_{\rho \rho}(\mathbf{x}, \tau)=\rho_{0} \frac{x_{1} x_{j} x_{k} x_{\ell}}{16 \pi^{2} c_{0}^{5} x^{6}} \iint_{\text {jet }} \frac{\partial^{4}}{\partial \tau^{4}} \mathbb{R}_{t j k l}\left(\mathbf{y}^{\prime}, \eta, \tau+\frac{\boldsymbol{\eta} \cdot \mathbf{x}}{c_{0} x}\right) \mathrm{d} \mathbf{y}^{\prime} \mathrm{d} \boldsymbol{\eta}
$$

où $\boldsymbol{\eta}$ est le vecteur de séparation $\mathbf{y}^{\prime \prime}-\mathbf{y}^{\prime}, \mathbf{x} \cdot \boldsymbol{\eta} / x c_{0}$ représente le temps de retard $\tau_{\mathrm{r}}$ enregistré au point d'observation $\mathbf{x}$, pour deux ondes acoustiques émises au même instant, aux deux points $\mathbf{y}^{\prime}$ et $\mathbf{y}^{\prime \prime}$ et $\mathbb{R}_{i j k \ell}$ est le tenseur de corrélation d'ordre quatre, en deux points $\mathbf{y}^{\prime}$ et $\mathbf{y}^{\prime \prime}$ et au temps $\tau$, défini par :

$$
\left.\mathbb{R}_{\imath, k \ell}\left(\mathbf{y}^{\prime}, \boldsymbol{\eta}, \tau\right)=\overline{u_{\imath}^{\prime} u_{j}^{\prime}\left(\mathbf{y}^{\prime}, t\right) u_{k}^{\prime \prime} u_{\ell}^{\prime \prime}\left(\mathbf{y}^{\prime \prime}, t+\tau\right.}\right)-\mathbb{R}_{i j k \ell}^{0}\left(\mathbf{y}^{\prime}, \boldsymbol{\eta}\right)
$$

Dans cette dernière expression $\mathbb{R}_{t, k \ell}^{0}$ est un tenseur arbitraire indépendant du temps que l'on choisit convenablement pour simplifier les calculs. Rappelons ici que le calcul de la fonction d'auto-corrélation $C_{\rho \rho}$ permet la détermination directe de l'intensité acoustique et de son spectre. En effet, l'intensité acoustique sera égale à $C_{\rho \rho}(\mathbf{x}, \tau=0)$ et le spectre $\tilde{I}_{\omega}(\mathbf{x})$ de 
l'intensité acoustique est obtenu à partir de la transformée de Fourier temporelle de $C_{\rho \rho}(\mathbf{x}, \tau):$

$$
\tilde{I}_{\omega}(\mathbf{x})=\frac{1}{2 \pi} \int_{-\infty}^{+\infty} C_{\rho \rho}(\mathbf{x}, \tau) \mathrm{e}^{\omega \tau} \mathrm{d} \tau
$$

Dans le paragraphe suivant, on montre dans quelle limite on peut négliger le temps de retard $\tau_{\mathrm{r}}$. Cette simplification nous permettra de poursuivre la réduction de l'expression de $C_{\rho \rho}(\mathbf{x}, \tau)$ en fonction des grandeurs turbulentes accessibles par les moyens actuels de simulation.

2.2 EFFET DE CONVECTION DES SOURCES ET INTRODUCTION D'UN RÉFÉRENTIEL MOBILE. - Soit $\ell$ une échelle typique de corrélation de la turbulence. Par définition, cette grandeur est telle que :

$$
\frac{\mathbb{R}_{l j k \ell}\left(\mathbf{y}^{\prime}, \boldsymbol{\eta}, \tau\right)}{\mathbb{R}_{l j k \ell}\left(\mathbf{y}^{\prime}, 0, \tau\right)} \approx 0 \text { pour }|\boldsymbol{\eta}|>\ell
$$

Si $\mathbb{R}_{i j k \ell}$ reste à peu près constant sur un intervalle de temps de l'ordre de $\ell / c_{0}$, c'est-à-dire si on considère un des tourbillons d'échelle $\ell$ ayant des temps caractéristiques $\tau_{\eta} \gg \ell / c_{0}$, il sera possible d'approcher $\mathbb{R}_{i j k \ell}\left(\mathbf{y}^{\prime}, \boldsymbol{\eta}, \tau+\frac{\mathbf{x} \cdot \boldsymbol{\eta}}{x \cdot c_{0}}\right)$ de l'équation (4) par $\mathbb{R}_{i j k \ell}\left(\mathbf{y}^{\prime}, \boldsymbol{\eta}, \tau\right)$. Autrement dit, on peut dans ce cas négliger le temps retard $\tau_{\mathrm{r}}=\mathbf{x} \cdot \boldsymbol{\eta} / x \cdot c_{0}$ au point d'observation $\mathbf{x}$. Or l'inégalité (6) n'est pas généralement satisfaite dans les écoulements réels à vitesse moyenne élevée. En effet, il faut tenir compte du phénomène de convection des structures turbulentes par l'écoulement moyen. Les structures turbulentes produisent des fluctuations très rapides par rapport à un observateur fixe ( $\tau_{\eta}$ sera très petit). Cependant, ces fluctuations vues par un observateur mobile se déplaçant avec l'écoulement sont beaucoup plus lentes. Ainsi la structure turbulente apparaîtra comme " gelée » dans un référentiel en mouvement à la vitesse de convection $U_{1 \mathrm{c}}$ des tourbillons (un résultat qui est souvent désigné sous le nom d'《 hypothèse de Taylor »).

En introduisant le repère mobile $\xi$ où $\xi=\eta-\hat{i} U_{1 \mathrm{c}} \tau$ (où î représente le vecteur unitaire dans la direction de l'écoulement moyen), on définit le tenseur de corrélation $R_{t j k \ell}$, dans le repère mobile $\boldsymbol{\xi}$, par :

$$
R_{\imath \jmath k \ell}\left(\mathbf{y}^{\prime}, \xi, \tau\right)=\mathbb{R}_{i j k \ell}\left(\mathbf{y}^{\prime}, \eta, \tau\right) .
$$

Le tenseur $R_{\imath j k \ell}$ peut être interprété comme étant le tenseur de corrélation vu par un observateur se déplaçant à la vitesse $U_{1 \mathrm{c}}$, mais qui mesure l'amplitude des fluctuations de vitesse relativement à un repère fixe. En introduisant ce changement de repère dans (4) et en négligeant les temps de retard, $C_{\rho \rho}(\mathbf{x}, \tau)$ s'écrit :

$$
C_{\rho \rho}(\mathbf{x}, \tau)=\frac{\rho_{0} x_{1} x_{j} x_{k} x_{\ell}}{16 \pi^{2} c_{0}^{5} x^{6}} \iint_{\text {jet }} \frac{1}{C^{5}}\left\{\frac{\partial^{4}}{\partial \tau^{4}} R_{t j k \ell}\left(\mathbf{y}^{\prime}, \xi, \tau^{\prime}\right)\right\}_{\tau^{\prime}=\frac{\tau}{C}} \mathrm{~d} \xi \mathrm{d} \mathbf{y}^{\prime}
$$

où $C=1-M_{\mathrm{c}} \cos \theta ; \cos \theta=x_{1} / x$.

Dans ces expressions, $C$ représente le facteur de convection et $M_{\mathrm{C}}$ le nombre de Mach de convection, $\theta$ est l'angle entre la direction de l'écoulement moyen et la direction correspondant au point d'observation $x$ (cf. Fig. 1). Notons que la puissance du facteur d'amplification $C$ est de -5 , correspondant au facteur d'amplification d'une source quadripolaire convectée à 




Fig. 1. - Configuration du jet turbulent. $(x, \theta, \phi$ coordonnées sphériques du point d'observation $\mathbf{x}$, $D$ le diamètre de la buse et $y$ le point milieu des 2 points sources $y^{\prime}$ et $\mathbf{y}^{\prime \prime}$ ).

[Turbulent jet configuration. ( $x, \theta, \phi$ spherical coordinates of point of observation $\mathrm{x}, D$ diameter of the nozzle and $y$ the midpoint of the two source points $y^{\prime}$ and $\left.y^{\prime \prime}\right)$.]

une vitesse $U_{1 \mathrm{c}}$ (Ffowcs Williams, [5]). En prenant la transformée de Fourier de la relation (8), le spectre de l'intensité acoustique $\tilde{I}_{\omega}(\mathbf{x})$ est le suivant :

$$
\tilde{I}_{\omega}(\mathbf{x})=\frac{\omega^{4} \rho_{0} x_{i} x_{j} x_{k} x_{\ell}}{32 \pi^{3} c_{0}^{5} x^{6}} \times \int_{-\infty}^{+\infty} \iint_{\text {jet }} \exp [+j \omega C \tau] R_{\imath \jmath k \ell}\left(\mathbf{y}^{\prime}, \xi, \tau\right) \mathrm{d} \xi \mathrm{d} \mathbf{y}^{\prime} \mathrm{d} \tau .
$$

D'après (9), on remarque qu'à l'émission acoustique de fréquence $\omega C / 2 \pi$ dans le repère mobile, correspond la fréquence $\omega / 2 \pi$ enregistrée en champ lointain dans le repère fixe. $\mathrm{Ce}$ facteur d'amplification, $1 / C$, est le même que celui de l'effet Doppler pour le cas idéal d'un point source se déplaçant à la vitesse $U_{1}$.

2.3 MODÈle DE RIBNER. - Dans ses deux premiers modèles [20, 21] Ribner réécrit la relation (8) sous la forme proposé par Proudman [19] où $\overline{u_{x}^{\prime 2} u_{x}^{\prime \prime 2}}$ gouverne le rayonnement acoustique dans la direction $\mathbf{x}$. Ce formalisme simplifie notablement l'analyse, mais il faut

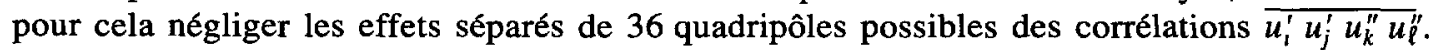
Dans son troisième modèle, Ribner [22] comble cette lacune. Toutes les corrélations

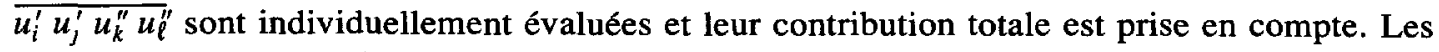
hypothèses de calcul de ce modèle sont les suivantes :

H1) Le jet considéré est axisymétrique et par suite la fonction d'auto-corrélation $C_{\rho \rho}$ est indépendante de $\phi$.

H2) L'écoulement moyen pour un jet axisymétrique est pratiquement parallèle, d'où la décomposition suivante de l'écoulement instantané en écoulement moyen parallèle et en écoulement turbulent tridimensionnel :

$$
u_{\imath}(\mathbf{y}, t)=U_{\imath}(\mathbf{y}) \delta_{1,}+u_{\mathrm{t} t}(\mathbf{y}, t)
$$


En introduisant cette décomposition dans $R_{t j k \ell}$ et en supposant que la turbulence considérée est homogène, le tenseur $R_{i j k l}$ se met sous la forme suivante :

$$
\begin{aligned}
R_{\imath \jmath k \ell}(\mathbf{y}, \boldsymbol{\xi}, \tau)=\overline{u_{\mathrm{t} i}^{\prime} u_{\mathrm{v}}^{\prime} u_{\mathrm{t} k}^{\prime \prime} u_{\mathrm{l} \ell}^{\prime \prime}}+U_{1}^{\prime} U_{1}^{\prime \prime}\left(\delta_{1,} \delta_{1 k} \overline{u_{\mathrm{t}}^{\prime} u_{\mathrm{t} \ell}^{\prime \prime}}+\right. \\
\left.+\delta_{1,} \delta_{1 \ell} \overline{u_{\mathrm{t} l}^{\prime} u_{\mathrm{t} k}^{\prime \prime}}+\delta_{1,} \delta_{1 k} \overline{u_{t l}^{\prime} u_{\mathrm{t} \ell}^{\prime \prime}}+\delta_{1,} \delta_{1 \ell} \overline{u_{\mathrm{t}}^{\prime} u_{\mathrm{l} k}^{\prime \prime}}\right) .
\end{aligned}
$$

Le premier terme représente la contribution au bruit due à la turbulence toute seule. Cette contribution est appelée «Bruit Propre». Les autres termes représentent par contre la contribution au bruit des termes d'interaction turbulence-écoulement moyen. On désigne cette contribution par « Bruit de Cisaillement » car on peut facilement montrer (Ribner, [21]) que la contribution de ces termes est nulle pour un écoulement moyen homogène.

H3) La loi de probabilité conjointe de la vitesse turbulente $u_{\mathrm{t}}$ est normale. Ainsi le terme de bruit propre peut être exprimé en fonction du tenseur de corrélation double $R_{\imath \jmath}(\mathbf{y}, \xi, \tau)$ :

$$
\overline{u_{t i}^{\prime} u_{\mathrm{t} j}^{\prime} u_{\mathrm{t} k}^{\prime \prime} u_{\mathrm{t} \ell}^{\prime \prime}}(\mathrm{y}, \xi, \tau)=R_{i j}(\mathbf{y}, 0,0) R_{k \ell}(\mathbf{y}, 0,0)+\left(R_{t k} R_{j \ell}+R_{\imath \ell} R_{j k}\right)(\mathbf{y}, \xi, \tau)
$$

où

$$
R_{\imath \jmath}(\mathbf{y}, \boldsymbol{\xi}, \tau)=\overline{u_{\mathfrak{u}}^{\prime} u_{\mathrm{y}}^{\prime}}(\mathbf{y}, \boldsymbol{\xi}, \tau)
$$

H4) En admettant que pour le tenseur double $R_{i j}(\mathbf{y}, \xi, \tau)$ les variables spatiales et temporelles sont séparables, que la turbulence considérée est aussi isotrope, et que les fonctions spatiales et temporelles ainsi introduites ont une forme gaussienne, ce tenseur devient :

$$
\begin{aligned}
R_{\imath \jmath}(\mathbf{y}, \xi, \tau) & =R_{t \jmath}(\mathbf{y}, \xi) \exp \left(-\omega_{\mathrm{f}}^{2} \tau^{2}\right) \\
R_{\imath \jmath}(\mathbf{y}, \boldsymbol{\xi}) & =\overline{u_{\mathrm{t} m}^{2}}\left[(f+1 / 2 \xi f \partial f / \partial \xi) \delta_{\imath \jmath}-1 / 2 \partial f / \partial \xi \xi_{\imath} \xi_{J} / \xi\right] \\
f(\xi) & =\exp \left(-\pi \xi^{2} / L_{1}^{2}\right)
\end{aligned}
$$

où $f$ est la fonction de corrélation longitudinale, $\omega_{f}$ une pulsation caractéristique de la turbulence, $L_{1}$ une échelle intégrale longitudinale et $\overline{u_{\mathrm{t} m}^{2}}$ représente $2 / 3$ de l'énergie cinétique turbulente $K$.

H5) Il reste à modéliser le terme défini en deux points $U_{1}^{\prime}\left(\mathbf{y}^{\prime}\right) U_{1}^{\prime \prime}\left(\mathbf{y}^{\prime \prime}\right)$ en fonction de $U_{1}^{2}(\mathbf{y})$. La modélisation proposée est une gaussienne de la forme $U_{1}^{2}(\mathbf{y}) \exp \left(-\sigma \pi \xi_{2}^{2} / L_{1}^{2}\right)$ où $\sigma$ est un coefficient local égal à 0,07 [Ribner, 22]. Cependant cette modélisation n'est pas bien adaptée. En effet, à partir d'un champ de vitesse connu à l'avance, nous avons recalculé le coefficient $\sigma$ pour divers points situés à $y_{1} / D=4$ et qui s'éloignent progressivement de l'axe $\left(y_{2} / D=0,0,51\right.$ et 3) [Béchara, 1]. Les calculs montrent d'une part que le coefficient $\sigma$ n'est pas constant et d'autre part, que l'écart pour $y_{2} / D \geqslant 1$, devient considérable entre le calcul exact de $U_{1}^{\prime}\left(\mathbf{y}^{\prime}\right) U_{1}^{\prime \prime}\left(\mathbf{y}^{\prime \prime}\right)$ et l'exponentielle en question.

Pour éviter cette modélisation, nous avons réalisé un développement limité à l'ordre 1 de $U_{1}^{\prime}\left(\mathbf{y}^{\prime}\right) U_{1}^{\prime \prime}\left(\mathbf{y}^{\prime \prime}\right)$ en fonction du point milieu $\mathbf{y}$ tel que :

$$
U_{1}^{\prime}\left(\mathbf{y}^{\prime}\right) U_{1}^{\prime \prime}\left(\mathbf{y}^{\prime \prime}\right)=U_{1}\left(\mathbf{y}-\xi_{2} / 2\right) U_{1}\left(\mathbf{y}+\xi_{2} / 2\right)=U_{1}^{2}(\mathbf{y})-\frac{\xi_{2}^{2}}{4}\left[\frac{\partial U_{1}}{\partial y_{2}}(\mathbf{y})\right]^{2}
$$

Dans ce qui précède, on a donc adopté les hypothèses initialement introduites par Ribner avec cependant une modification de la modélisation du produit $U_{1}^{\prime} U_{1}^{\prime \prime}$ des vitesses axiales en deux points. 
En tenant compte de toutes ces hypothèses, et après transformation de la relation (8), les expressions de l'intensité acoustique directionnelle par unité de volume du jet pour le bruit propre et de cisaillement sont les suivantes :

$$
\begin{gathered}
I^{\mathrm{BP}}(x, \theta \mid \mathbf{y})=\frac{3 \sqrt{2} \rho_{0} L_{1}^{3}{\overline{u_{\mathrm{t} m}^{2}}}^{2}}{4 \pi^{2} c_{0}^{5} x^{2}} \omega_{\mathrm{f}}^{4} \frac{D_{l}^{\mathrm{BP}}}{C^{5}} \\
I^{\mathrm{BC}}(x, \theta \mid \mathbf{y})=\frac{3}{8} \frac{\rho_{0} L_{1}^{5} \overline{u_{\mathrm{t} m}^{2}}}{\pi^{3} c_{0}^{5} x^{2}}\left(\frac{\partial U_{1}}{\partial y_{2}}\right)^{2} \omega_{\mathrm{f}}^{4} \frac{D_{l}^{\mathrm{BC}}}{C^{5}}
\end{gathered}
$$

où $D_{i}^{\mathrm{BC}}=1 / 2\left(\cos ^{2} \theta+\cos ^{4} \theta\right)$ est la directivité intrinsèque du bruit de cisaillement. Par contre, le calcul montre que la directivité intrinsèque du bruit propre $D_{l}^{\mathrm{BP}}$ est égale à l'unité. Cette indépendance vis-à-vis de l'angle d'observation $\theta$ découle simplement de l'hypothèse de l'isotropie de la turbulence. Notons que l'expression (17) du bruit de cisaillement est plus adaptée que celle développée par Ribner [22] :

$$
I^{\mathrm{BC}}(x, \theta \mid \mathbf{y})=\frac{3}{2} \frac{\rho_{0} L_{1}^{3} \overline{u_{\mathrm{t} m}^{2}}}{\pi^{2} c_{0}^{5} x^{2}} U_{1}^{2} \frac{\sigma}{(1+\sigma)^{3 / 2}} \omega_{\mathrm{f}}^{4} \frac{D_{l}^{\mathrm{BC}}}{C^{5}}
$$

En effet, dans cette expression, en plus des réserves déjà signalées (voir H5), l'intensité du bruit est proportionnelle au carré de vitesse longitudinale $U_{1}$ et ne s'annule pas pour un écoulement moyen uniforme.

L'intensité acoustique directionnelle, du bruit total s'exprime simplement comme la somme des deux contributions du bruit propre et du bruit de cisaillement :

$$
I(x, \theta \mid \mathbf{y})=I^{\mathrm{BP}}(x, \theta \mid \mathbf{y})+I^{\mathrm{BC}}(x, \theta \mid \mathbf{y}) .
$$

Nous pouvons noter que cette intensité totale peut se mettre sous la forme :

$$
\left[A+\quad B / 2\left(\cos ^{4} \theta+\cos ^{2} \theta\right)\right] \quad 1 / C^{5}
$$

Bruit propre Bruit de cisaillement Effet de convection

La contribution du bruit propre est omnidirectionnelle, alors que celle du bruit de cisaillement est semblable à la contribution d'un dipôle. La combinaison de ces deux contributions pour $A=B=1$ est quasiment une ellipse, dont le grand axe est parallèle à la direction de l'écoulement. L'amplification correspondant à cet axe sera de $3 \mathrm{~dB}$. Quant à l'effet de convection, le facteur $1 / C^{5}$ tend largement à amplifier l'intensité pour les angles aigus et à atténuer pour les angles obtus ; et cette amplification devient plus conséquente avec des valeurs de nombres de Mach de convection $M_{c}$ élevées. Pour éviter une amplification excessive à ces nombres de Mach, une analyse plus fine de Ribner [21, 22] et de Ffowcs Williams [5] montre l'intérêt d'utiliser un facteur de convection modifié de la forme :

$$
C_{\mathrm{m}}=\left[\left(1-M_{\mathrm{c}} \cos \theta\right)^{2}+\alpha^{2} M_{\mathrm{c}}^{2}\right]^{1 / 2}
$$

où $\alpha M_{\mathrm{c}}=\omega_{\mathrm{f}} L_{1} /\left(\sqrt{\pi} c_{0}\right)$. Selon Ribner [22], $\alpha$ a pour valeur 0,55 alors que pour Davies $e t$ al. [4], la constante $\alpha$ est égale à 0,3 .

2.4 MODÈle DE GOLDSTEIN. - Ce modèle est une généralisation de celui proposé par Ribner. L'hypothèse d'une turbulence isotrope est remplacée par celle d'une turbulence axisymétrique. En effet, l'écoulement moyen introduit une direction privilégiée et l'hypothèse d'une 
turbulence isotrope [16] néglige des effets importants d'anisotropie comme l'étirement des structures turbulentes et la compression transversale de ces structures par l'écoulement. Ainsi, les grandes structures ont tendance à devenir des structures cylindriques dont l'échelle longitudinale vaut approximativement trois fois l'échelle transversale [8, 4].

La démarche suivie par Goldstein est similaire à celle de Ribner, et nous nous contentons ici de rappeler les différentes expressions de l'intensité acoustique directionnelle pour le bruit propre et de cisaillement :

$$
I^{\mathrm{BP}}(x, \theta \mid y)=\rho_{0} \frac{12 L_{1} L_{2}^{2}}{5 \pi c_{0}^{5} x^{2}} \bar{u}_{t 1}^{2} \omega_{\mathrm{f}}^{4} \frac{D_{l}^{\mathrm{BP}}}{C^{5}}
$$

où

$$
\begin{gathered}
D_{i}^{\mathrm{BP}}=\left\{1+2(M / 9-N) \cos ^{2} \theta \sin ^{2} \theta+\right. \\
\left.+1 / 3\left[M^{2} / 7+M-1,5 N\left(3-3 N+1,5 / \Delta^{2}-\Delta^{2} / 2\right)\right] \sin ^{4} \theta\right\} \\
I^{\mathrm{BC}}(x, \theta \mid \mathrm{y})=\rho_{0} \frac{24 L_{1} L_{2}^{4} \overline{u_{\mathrm{t}}^{2}}}{\pi c_{0}^{5} x^{2}}\left(\frac{\partial U_{1}}{\partial y_{2}}\right)^{2} \omega_{\mathrm{f}} \frac{D_{1}^{\mathrm{BC}}}{C^{5}}
\end{gathered}
$$

où

$$
D_{i}^{\mathrm{BC}}=\cos ^{2} \theta\left(\cos ^{2} \theta+\frac{1}{2}\left[1 / \Delta^{2}-2 N\right] \sin ^{2} \theta\right)
$$

Dans ces expressions :

$$
\begin{aligned}
& \Delta=L_{2} / L_{1} \\
& N=1-\overline{u_{\mathfrak{t} 2}^{2}} / \overline{u_{\mathrm{t} 1}^{2}}
\end{aligned}
$$

et

$$
M=[1,5(\Delta-1 / \Delta)]^{2}
$$

$L_{1}$ et $\overline{u_{\mathrm{t} 1}^{2}}$ (respectivement $L_{2}$ et $\overline{u_{\mathrm{t} 2}^{2}}$ ) sont l'échelle intégrale et l'énergie cinétique longitudinales (respectivement transversales) de turbulence. A l'aide des paramètres $\Delta, M$ et $N$, les relations (21a et c) expriment l'effet de l'anisotropie des structures turbulentes sur les directivités intrinsèques du bruit propre $D_{l}^{\mathrm{BP}}$ et de cisaillement $D_{l}^{\mathrm{BC}}$

Si nous exprimons l'intensité acoustique totale sous une forme semblable à (19), nous remarquons que la contribution du bruit propre est directionnelle. Elle est analogue à celle d'un dipôle dont l'axe est perpendiculaire à la direction de l'écoulement. C'est une différence fondamentale par rapport à la directivité intrinsèque uniforme du bruit propre de Ribner. Par contre, la directivité du bruit de cisaillement garde une forme dipolaire proche de celle de Ribner. D'autre part, les relations (21b et d) montrent que pour $\Delta=1$ et $N=0$ qui correspondent à une turbulence isotrope, on retrouve ainsi les directivités de Ribner.

\section{Détermination des grandeurs aérodynamiques.}

Pour réaliser un calcul numérique de l'intensité acoustique directionnelle par unité de volume $I(x, \theta \mid y)$, les relations du modèle de Ribner $(16,17)$ et Goldstein $(21 \mathrm{a}, 21 \mathrm{c})$ supposent que l'on connaît localement les grandeurs suivantes :

$\frac{U_{1}}{u_{\mathrm{t} m}^{2}}, \overline{u_{\mathrm{t} 1}^{2}}$ et $\overline{u_{\mathrm{t} 2}^{2}}$ vitesse axiale de l'écoulement moyen, énergie cinétique turbulente moyenne, longitudinale et transversale, 
$L_{1}$ et $L_{2} \quad$ échelle intégrale longitudinale et transversale de turbulence,

$\omega_{\mathrm{f}} \quad$ pulsation caractéristique de turbulence,

$U_{1 \text { c }} \quad$ vitesse axiale moyenne de convection.

Les grandeurs $U_{1}$ et $\overline{u_{\mathrm{tm}}^{2}}$ sont directement obtenues à partir d'une résolution numérique des équations de Navier-Stokes moyennées utilisant un modèle de turbulence à deux équations de transport de type $K-\varepsilon$. Cette résolution est effectuée avec le code ULYSSE développé par le Département Laboratoire National d'Hydraulique de la Direction des Etudes et Recherches d'Electricité de France [13]. La version standard du modèle $K-\varepsilon$ [10a] est utilisée dans ces calculs avec les valeurs classiques des constantes $\left(C_{\mu}=0,09, C_{\varepsilon 1}=1,44, C_{\varepsilon 2}=1,92\right.$, $\sigma_{k}=1, \sigma_{\varepsilon}=1,3$ ). L'algorithme du code (elliptique en espace et parabolique en temps) est basé sur la technique des pas de temps fractionnaires permettant de définir plusieurs sous étapes et d'utiliser des solveurs adaptés aux propriétés de chaque sous-étape ainsi définie. En particulier, la sous-étape de convection est résolue avec la méthode des caractéristiques et celle de diffusion par une méthode de type résidu conjugué à trois couches. Des tests de validation [1] sont réalisés en comparant les résultats numériques de $U_{1}$ et $\overline{u_{\mathrm{t} m}^{2}}$ avec les données de Pao [18] et Davies et al. [4]. Ces tests montrent qu'on retrouve bien les profils radiaux « autosemblables » de $U_{1}$ et $\sqrt{\overline{u_{t m}^{2}}}$, exprimés en variables réduites dans les zones de mélange et développée du jet.

Pour les grandeurs restantes, on utilise les relations de fermetures suivantes :

i) d'après le concept de viscosité turbulente $\nu_{t}$, les deux énergies turbulentes $\overline{u_{t l}^{2}}$ et $\overline{u_{12}^{2}}$ sont exprimées par :

$$
\begin{aligned}
& \overline{u_{\mathrm{t} 1}^{2}}=-2 \nu_{\mathrm{t}} \partial U_{1} / \partial y_{1}+2 / 3 K \\
& \overline{u_{\mathrm{t} 2}^{2}}=-2 \nu_{\mathrm{t}} \partial U_{2} / \partial y_{2}+2 / 3 K
\end{aligned}
$$

où $\nu_{\mathrm{t}}=C_{\mu} K^{2} / \varepsilon$ est la viscosité cinématique turbulente et $\varepsilon$ est le taux de dissipation de l'énergie cinétique turbulente.

Le calcul numérique montre que $\overline{u_{\mathrm{t} 1}^{2}} \cong \overline{u_{\mathrm{t} 2}^{2}} \cong 2 / 3 \mathrm{~K}$. Ceci est lié au fait que le concept de viscosité turbulente représente de façon imparfaite la répartition de l'énergie turbulente selon les composantes 1 et 2 ( $\mathrm{Ha}$ Minh [8a]).

ii) On peut définir les échelles intégrales de turbulence longitudinale et transversale de la manière suivante :

$$
L_{1}={\overline{u_{\mathrm{t} 1}^{2}}}^{3 / 2} / \varepsilon \quad \text { et } \quad L_{2}={\overline{u_{\mathrm{t} 2}^{2}}}^{3 / 2} / \varepsilon .
$$

Comme les composantes $\overline{u_{\mathrm{t} 1}^{2}}$ et $\overline{u_{\mathrm{t} 1}^{2}}$ sont quasi isotropes, l'échelle de turbulence $L_{2} \mathrm{~s}$ 'identifie à $L_{1}$. Or les données expérimentales montrent que le rapport $\Delta=L_{2} / L_{1}$ est différent de l'unité (ainsi par exemple $\Delta=1 / 3$ d'après Davies et al. [4]). Il paraît donc nécessaire d'imposer cette valeur au rapport $\Delta$ pour éviter que la directivité intrinsèque du bruit total du modèle de Goldstein ne se réduise à celle du modèle de Ribner (voir $21 \mathrm{~b}$ ). Cette valeur est celle qui donne les meilleurs résultats sur une large gamme de vitesses subsoniques même si pour des vitesses faibles, le résultat est un peu moins satisfaisant.

iii) La modélisation la plus naturelle consiste à exprimer $\omega_{\mathrm{f}}$ en fonction de la fréquence caractéristique donnée par $\varepsilon / K[2]$ :

$$
\omega_{\mathrm{f}}=2 \pi \varepsilon / K
$$


Notons que la modélisation des échelles de turbulence $L_{1}$ et $L_{2}$ et de la pulsation $\omega_{\mathrm{f}}$ par les relations $(23$ et 24$)$ est réalisée à un facteur de proportionnalité près. Comme on ne dispose pas de données expérimentales fiables pour déterminer ces facteurs, il faut introduire un facteur de calage global dans l'expression de l'intensité acoustique directionnelle pour chacun des modèles de Ribner et Goldstein ( $F_{\mathrm{C}}^{\mathrm{R}}$ et $F_{\mathrm{C}}^{\mathrm{G}}$ respectivement). La détermination de ces facteurs est abordée dans la section suivante.

iv) La vitesse de convection $U_{1 \text { c }}$ caractérisant la vitesse de convection des structures turbulentes ne peut être déterminée à partir du calcul aérodynamique et des grandeurs turbulentes $K-\varepsilon$. En général, on considère que cette vitesse est constante dans tout le domaine du jet et la valeur attribuée à cette constante est de l'ordre de 0,6 à 0,7 fois la vitesse de sortie du jet. Pour affiner cette modélisation, on adopte pour les 2 modèles, le profil expérimental de la vitesse de convection, en variables réduites $U_{1 \mathrm{c}} / U_{1 \mathrm{r}}$, mesuré par Davies et al. [4] dans une zone de mélange turbulente. On suppose en outre, que ce profil reste valable dans le jet, et ainsi déduire les valeurs locales de $U_{1 \mathrm{c}}$. Il est à noter que, pour la zone de mélange, $U_{1 \mathrm{r}}$ est égale à la vitesse de sortie du jet $U_{1 \mathrm{~b}}$ et, pour la zone de transition et la zone développée, $U_{1 \mathrm{r}}$ est identifiée à la vitesse axiale $U_{1}$ sur l'axe (pour $y_{2}=0$ ). On montre dans la section suivante l'amélioration apportée par cette modélisation par rapport à celle où on suppose $U_{1 \mathrm{c}}=$ Cte.

\section{Détermination des grandeurs acoustiques et comparaison avec des données expérimentales de Lush.}

Lush [17] présente des mesures acoustiques effectuées dans une chambre anéchoïque $(9 \times 9 \times 7,2 \mathrm{~m})$ pour le cas d'un jet d'air subsonique à température ambiante. La tuyère utilisée est convergente et a pour diamètre $D=25 \mathrm{~mm}$. Le microphone est placé sous différents angles par rapport à l'axe du jet en utilisant une structure en acier léger comportant un quadrant de cercle de rayon $3 \mathrm{~m}$, dont le centre coïncide avec le centre de la tuyère. Les angles de mesure varient de $7,5^{\circ}$ à $105^{\circ}$ avec un pas de $7,5^{\circ}$. Beaucoup de soins ont été pris pour s'assurer d'une part, que le bruit généré en amont de la tuyère de sortie est négligeable devant le bruit du jet et d'autre part que les réflexions sur le support du microphone sont minimales.

Pour chaque position angulaire, et pour chaque vitesse $U_{1 \mathrm{~b}}$ de sortie du jet qui variait entre 75 et $300 \mathrm{~m} / \mathrm{s}$ avec un intervalle de $22,5 \mathrm{~m} / \mathrm{s}, l^{\prime}$ intensité acoustique directionnelle $I(x, \theta)$ a été mesurée. En intégrant cette intensité par rapport à $\theta$, on peut obtenir la puissance acoustique totale du jet $W$. Les deux expressions reliant l'intensité acoustique directionnelle et par unité de volume à la puissance acoustique totale $W$ sont les suivantes :

$$
\begin{gathered}
I(x, \theta)=\int_{\mathrm{jet}} I(x, \theta \mid y) \mathrm{d} y \\
W=2 \pi x^{2} \int_{\theta_{\min }}^{\theta_{\max }} I(x, \theta) \sin \theta \mathrm{d} \theta \\
\theta_{\min }=7,5^{\circ} ; \quad \theta_{\max }=105^{\circ}
\end{gathered}
$$

où

Théoriquement, la puissance acoustique doit être intégrée entre 0 et $180^{\circ}$. Or comme 1 'a souligné Lush [17], $I(x, \theta \mid y)$ est atténuée largement par le facteur de convection pour des angles obtus (Sect. 2.3) et par le facteur $\sin \theta$ pour les angles proches de $0^{\circ}$. Ainsi l'approximation qui consiste à limiter l'intervalle de mesure de $\left[\theta_{\min }, \theta_{\max }\right]$ à $\left[7,5^{\circ}, 105^{\circ}\right]$ introduit une erreur négligeable. 
Avant de comparer ces données expérimentales avec les calculs numériques, nous allons tout d'abord déterminer la configuration optimale pour chacun des deux modèles ainsi que les facteurs de calage $F_{\mathrm{C}}^{\mathrm{R}}$ et $F_{\mathrm{C}}^{\mathrm{G}}$.

4.1 DÉtermination de la CONFIGURATION optimale ET DES FACTEURS DE CALAGE. - Dans la section 2.3, nous avons rappelé la nécessité d'introduire une correction $\alpha M_{\mathrm{c}}$ au facteur de convection $C$ pour des vitesses élevées (relation 20). Comme plusieurs choix sont possibles pour calculer $\alpha$ et $M_{c}$, il est nécessaire de déterminer le choix optimal. D'autre part, il faut déterminer les facteurs de calage $F_{\mathrm{C}}^{\mathrm{R}}$ et $F_{\mathrm{C}}^{\mathrm{G}}$ pour les 2 modèles introduits par la modélisation des grandeurs $\omega_{\mathrm{f}}, L_{1}$ et $L_{2}$ à un facteur de proportionalité près. La procédure est la suivante :

pour chacun des deux modèles et pour un choix donné de $\alpha$ et $U_{1 \mathrm{c}}$, le facteur de calage global est calculé de telle sorte que l'intensité acoustique directionnelle $I\left(x, \theta=90^{\circ}\right)$ coïncide avec la valeur expérimentale de Lush pour une vitesse de sortie de $125 \mathrm{~m} / \mathrm{s}$. On calcule ensuite, l'écart $E_{\mathrm{p}}$ entre la puissance acoustique totale $W$ mesurée par Lush et celle calculée pour la vitesse de $300 \mathrm{~m} / \mathrm{s}$. La configuration optimale correspondra à l'écart $E_{\mathrm{p}}$ minimal, Le tableau I présente les cinq choix traités, avec les valeurs correspondantes de $F_{\mathrm{C}}$ et $E_{\mathrm{p}}$ pour les 2 modèles. Les calculs montrent que le choix optimal correspond au groupe de règles $C_{5}$ où l'écart

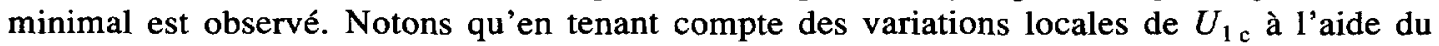

Tableau I. - Valeurs du facteur de calage $F_{\mathrm{C}}$ pour les 2 modèles correspondant à 5 groupes de règles différentes.

[Values of the adjustment factor $F_{\mathrm{C}}$ for the 2 models relative to 5 different groups of rules.]

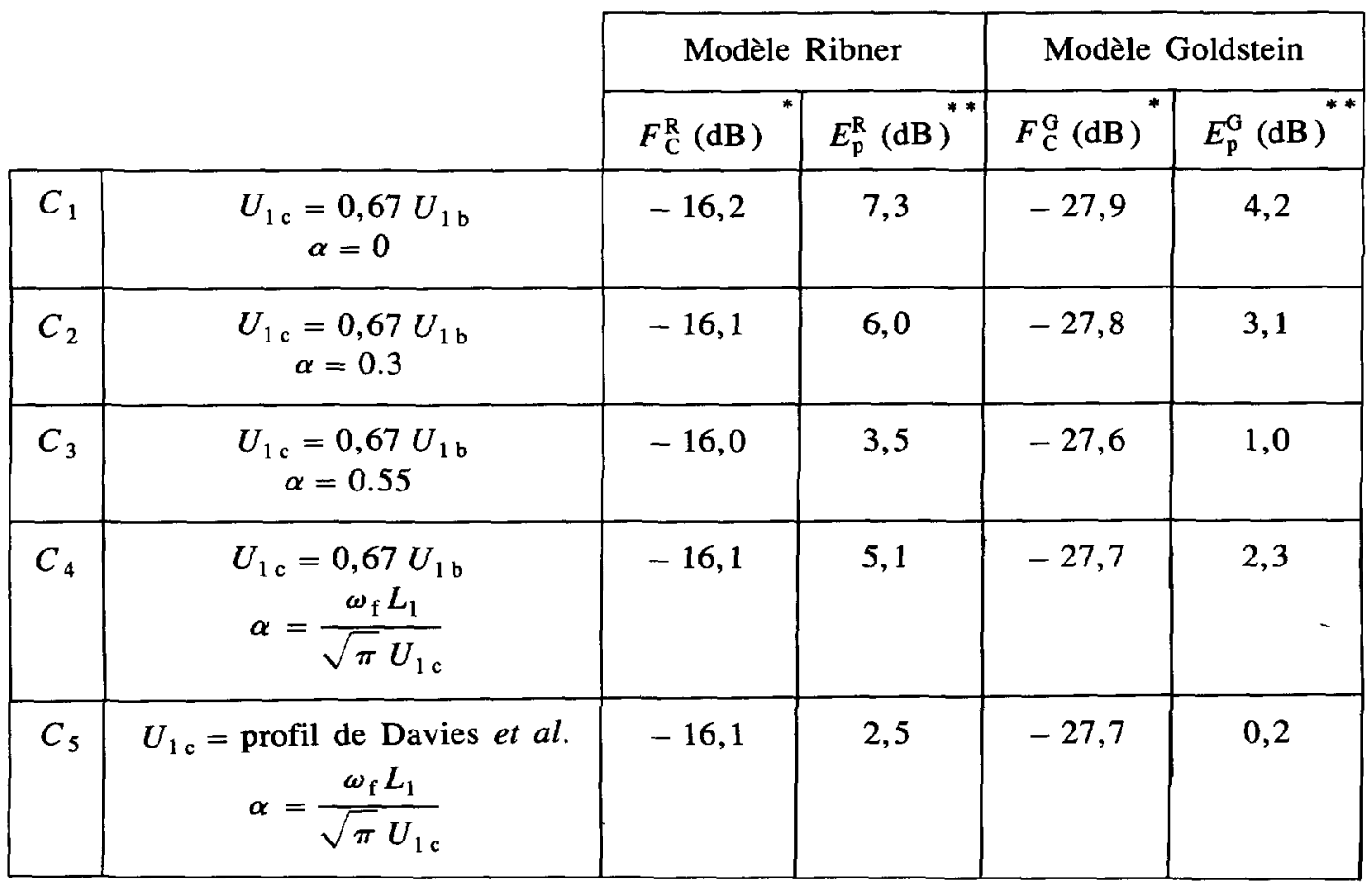

(*) $F_{\mathrm{C}}=I(x, \theta=90)_{\text {Lush }}-I(x, \theta=90)_{\text {code }}$ à $125 \mathrm{~m} / \mathrm{s}$.

(**) $E_{\mathrm{p}}=W_{\text {Lush }}-W_{\text {code }}$ à $300 \mathrm{~m} / \mathrm{s}$. 
profil de Davies comme suggéré à la section 3,1 'écart $E_{\mathrm{p}}$ du groupe $\mathrm{C}_{4}$ diminue au moins de $2 \mathrm{~dB}$ pour les 2 modèles comme le montre les règles $\mathrm{C}_{5}$.

Ainsi, tous les calculs que l'on va présenter ultérieurement correspondent au $5^{\mathrm{e}}$ groupe de règles de modélisation. Les 2 modèles ainsi adaptés sont respectivement désignés par $R_{\mathrm{a}}$ et $G_{\mathrm{a}}$.

4.2 VARIATION DE LA PUISSANCE ACOUSTIQUE EN FONCTION DE LA VITESSE DE SORTIE. - Le calcul numérique de la puissance acoustique est réalisé pour quatre vitesses de sortie de jet : $90,125,195$ et $300 \mathrm{~m} / \mathrm{s}$. La figure 2 montre que le modèle $G_{\mathrm{a}}$ reproduit exactement l'évolution de cette puissance comparée aux données expérimentales de Lush [17]. L'écart de puissance $E_{\mathrm{p}}$ à $300 \mathrm{~m} / \mathrm{s}$ est de $0,2 \mathrm{~dB}$, tandis que cet écart atteint $2,5 \mathrm{~dB}$ pour le modèle Ribner.

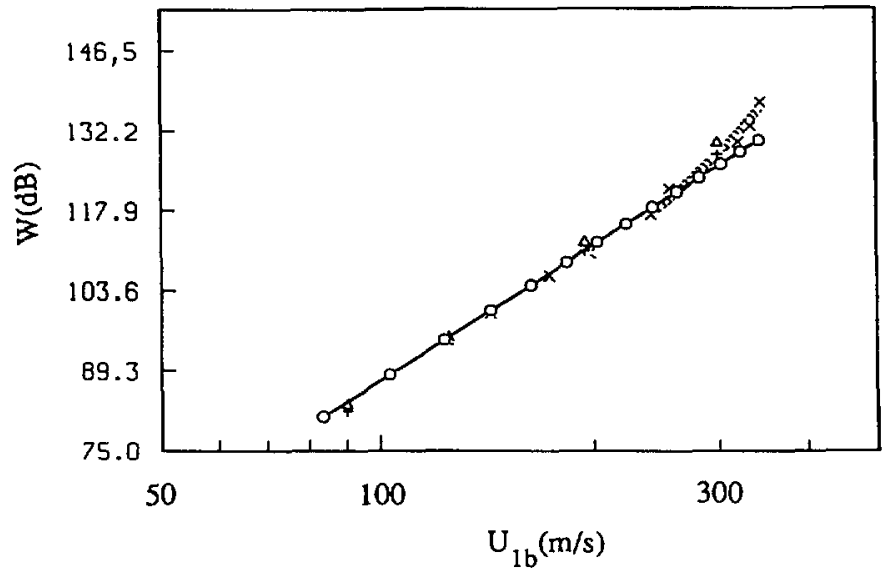

Fig. 2. - Variation de la puissance acoustique totale $W$ en fonction de la vitesse de sortie du jet $U_{1 \mathrm{~b}}$. ((O) Loi en $U_{1 \mathrm{~b}}^{8},(\triangle)$ modèle $R_{\mathrm{a}},(+)$ modèle $G_{\mathrm{a}},(\times)$ Lush, $(\diamond)$ Relation (27)).

[Variation of the total acoustic power $W$ as a function of jet exit velocity $U_{\mathrm{tb}} \cdot\left((O) U_{1 \mathrm{~b}}^{8}\right.$ law, $(\triangle)$ $R_{\mathrm{a}}$ model, (+) $G_{\mathrm{a}}$ model, ( $\times$ ) Lush $(\diamond)$ Relation (27)).]

D'autre part, en traçant sur la même figure la loi usuelle de variation de la puissance acoustique en $U_{1 b}^{8}$, on remarque que cette loi caractérise correctement l'évolution de la puissance acoustique pour des vitesses inférieures à $250 \mathrm{~m} / \mathrm{s}$. Au-delà de cette vitesse, il apparaît que les points expérimentaux évoluent avec une loi de pente supérieure à 8 . En fait, à ces vitesses élevées, l'effet de convection des sources devient important. En incluant cet effet, l'équivalent de la relation dimensionnelle en $U_{1 \mathrm{~b}}^{8}$ apparaît sous la forme donnée par Lush [17] :

$$
W \sim \rho_{0} U_{1 \mathrm{~b}}^{8} \frac{D^{2}}{c_{0}^{5}} \frac{1+M_{\mathrm{c}}^{2}}{\left(1-M_{\mathrm{c}}^{2}\right)^{4}} .
$$

En traçant la loi donnée par (27) pour $M_{\mathrm{c}}=0,67 U_{1 \mathrm{~b}} / c_{0}$, on retrouve l'évolution des points expérimentaux pour les vitesses supérieures à $250 \mathrm{~m} / \mathrm{s}$ (cf. Fig. 2). Notons que pour être cohérent avec les données de Lush [17], tous les calculs de puissances acoustiques exprimées en $\mathrm{dB}$ ont pour référence $10^{-13}$ Watt alors que celle des intensités acoustiques est de $10^{-12} \mathrm{Watt} / \mathrm{m}^{2}$. 
4.3 VARIATION DE L'INTENSITE ACOUSTIQUE DIRECTIONNELLE EN FONCTION DE L'ANGLE D'OBSERVATION $\theta$. - La relation (26) nous permet de calculer l'intensité acoustique $I(x, \theta)$ due à la totalité du jet pour un angle d'observation donné $\theta$. Les figures $3 \mathrm{a}, \mathrm{b}$ et $\mathrm{c}$ montrent la variation de cette intensité en fonction de $\theta$ pour les 2 modèles comparés aux données de Lush [17]. Pour les trois vitesses 125,195 et $300 \mathrm{~m} / \mathrm{s}$, le modèle $R_{\mathrm{a}}$ surestime $I(x, \theta)$ aux petits angles, et cette surestimation devient plus importante avec l'augmentation de la vitesse. Par contre le modèle $G_{\mathrm{a}}$ prévoit convenablement $l(x, \theta)$ aux petits angles pour les deux vitesses 195 et $300 \mathrm{~m} / \mathrm{s}$ et sous-estime l'intensité pour $125 \mathrm{~m} / \mathrm{s}$. Toutefois, il faut noter que le résultat obtenu par le modèle $G_{\mathrm{a}}$ à $300 \mathrm{~m} / \mathrm{s}$ est le plus correct (à notre connaissance) quand il est comparé à d'autres modèles basés sur la théorie de Lighthill utilisés dans la littérature. Pour permettre cette comparaison la figure $3 \mathrm{~d}$ montre l'évolution de $I(x, \theta)$ en

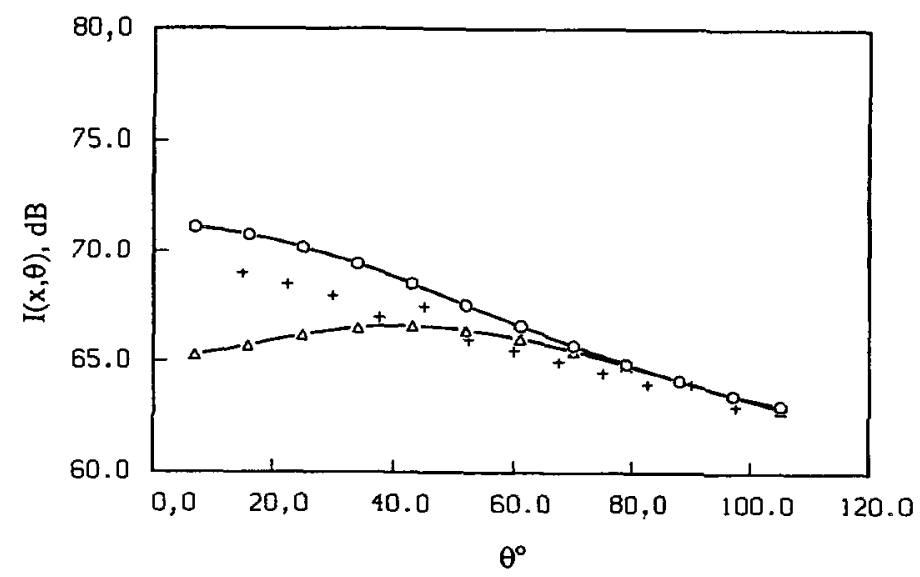

a)

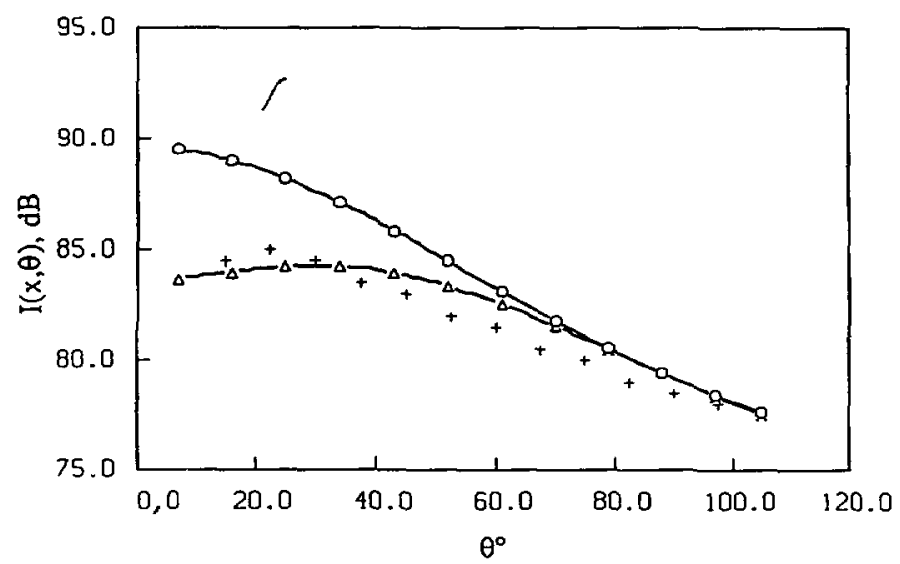

b)

Fig. 3. - Variation de l'intensité acoustique directionnelle du bruit total en fonction de l'angle d'observation $\theta$ pour les deux modèles $R_{\mathrm{a}}$ et $G_{\mathrm{a}}$. a) $125 \mathrm{~m} / \mathrm{s}$, b) $195 \mathrm{~m} / \mathrm{s}$, c) $300 \mathrm{~m} / \mathrm{s}$, d) $300 \mathrm{~m} / \mathrm{s}$. $((O)$ modèle $R_{\mathrm{a}},(\Delta)$ modèle $G_{\mathrm{a}},(+)$ Lush, $(\times)$ modèle simplifié, $(\diamond)$ modèle Bilanin, $(\nabla)$ modèle Hecht)).

[Variation of the acoustic directional intensity of total noise as a function of the angle of observation $\theta$ for the two models $R_{\mathrm{a}}$ and $G_{\mathrm{a}}$. a) $125 \mathrm{~m} / \mathrm{s}$, b) $195 \mathrm{~m} / \mathrm{s}$, c) $300 \mathrm{~m} / \mathrm{s}$, d) $300 \mathrm{~m} / \mathrm{s}$. ((O) $R_{\mathrm{a}}$ model, $(\Delta)$ $G_{\mathrm{a}}$ model, $(+)$ Lush $(\times)$ simplified model, $(\diamond)$ Bilanin model, $(\nabla)$ Hecht model)).] 


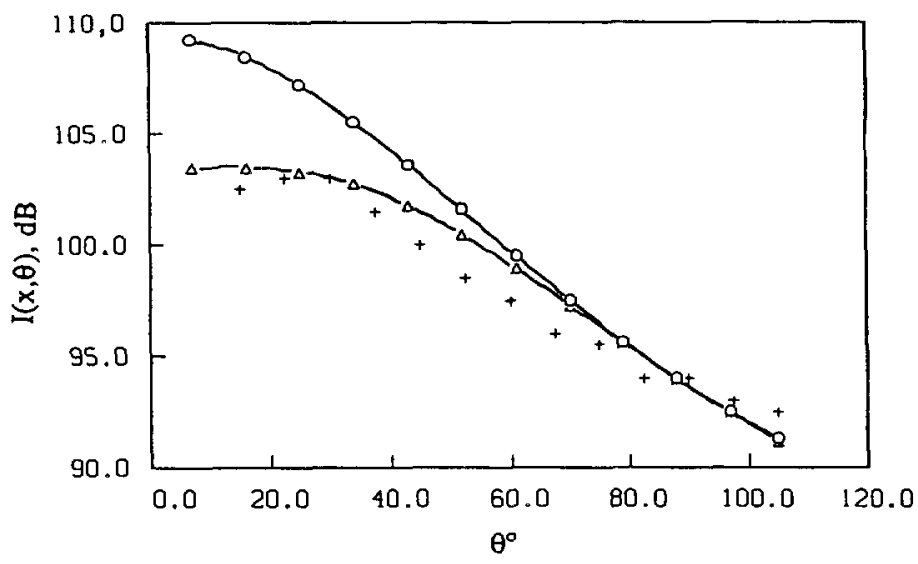

c)

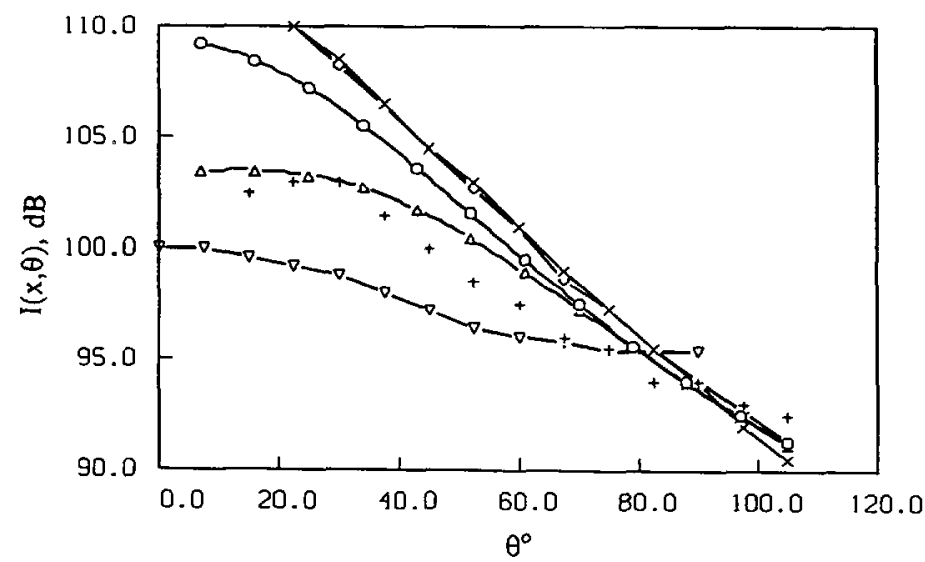

d)

Fig. 3 (suite).

fonction de $\theta$ pour les modèles $R_{\mathrm{a}}$ et $G_{\mathrm{a}}$ conjointement aux 3 modèles suivants :

1. Modèle simplifiée. - C'est le modèle basé sur l'analyse dimensionnelle où l'on tient compte de la convection des sources.

$$
I(x, \theta) \sim \rho_{0} \frac{U_{1 \mathrm{~b}}^{8}}{c_{0}^{5}} \frac{D^{2}}{x^{2}} \frac{1}{\left(1-M_{\mathrm{c}} \cos \theta\right)^{5}}
$$

où $M_{\mathrm{c}}=0,65 U_{1 \mathrm{~b}} / c_{0}$. Ce modèle est utilisé par Lush [17] pour comparaison avec ses données expérimentales.

2. Modèle de Bilanin et al. [3]. - Basé sur le $2^{e}$ modèle de Ribner [21] avec une modélisation du terme $U_{1}^{\prime} U_{1}^{\prime \prime}$ similaire à la notre. L'écoulement moyen et les grandeurs turbulentes sont calculées à partir d'un code de turbulence de type $K-\ell$ où $\ell$ est une longueur de mélange.

3. Modèle de Hecht et al. [9]. - Basé sur la détermination directe du tenseur de corrélation en deux points en deux temps $R_{l j}(y, \tau)$ à partir de la résolution de l'équation gouvernant ce 
tenseur dans le cas d'une turbulence homogène à cisaillement uniforme dans une seule direction.

Cette figure montre que le modèle de Bilanin a le même comportement que le modèle simplifié. Evidemment, le modèle de Ribner modifié basé sur un développement plus fin apporte une légère amélioration. De même, le modèle de Hecht qui est limité à un cisaillement uniforme, et qui utilise plusieurs lois de fermeture non validées pour la résolution numérique de l'équation gouvernant l'évolution de $R_{l}(y, \tau)$, ne donne pas en fin de compte une bonne prévision de la variation de $I(x, \theta)$ pour les petits angles $\theta$.

4.4 VARIATION DE L'INTENSITÉ ACOUSTIQUE DIRECTIONNELLE DE BRUIT PROPRE DE CISAILLEMENT EN FONCTION DE $\theta$. - Pour analyser plus finement la variation de l'intensité acoustique directionnelle $I(x, \theta)$ en fonction de $\theta$, nous avons calculé les deux contributions à cette intensité dues au bruit propre et de cisaillement. Les deux relations utilisées sont les suivantes :

$$
\begin{aligned}
I^{\mathrm{BP}}(x, \theta) & =\int_{\text {jet }} I^{\mathrm{BP}}(x, \theta \mid \mathbf{y}) \mathrm{d} \mathbf{y} \\
I^{\mathrm{BC}}(x, \theta) & =\int_{\text {jet }} I^{\mathrm{BC}}(x, \theta \mid \mathrm{y}) \mathrm{d} \mathbf{y}
\end{aligned}
$$

où $I^{\mathrm{BP}}$ et $I^{\mathrm{BC}}(x, \theta \mid \mathrm{y})$ sont données par les relations (16 et 17) et (21a et c) respectivement pour les deux modèles $R_{\mathrm{a}}$ et $G_{\mathrm{a}}$. Sur les figures 4 et 5 , on voit que l'intensité du bruit propre pour les deux modèles se confond avec celle du bruit total à $90^{\circ}$. Ceci est dû à ce que la directivité du bruit de cisaillement est fonction de $\cos \theta$. Pour le modèle $R_{\mathrm{a}} l^{\prime}$ 'intensité acoustique du bruit propre, ayant à l'origine une directivité intrinsèque isotrope, montre une amplification aux petits angles due au facteur de convection $1 / C^{5}$. En ajoutant le bruit de cisaillement qui est non négligeable pour $\theta<45^{\circ}$, cette amplification devient plus importante et ainsi ce modèle surestime l'intensité aux petits angles (cf. Fig. 4).

Par contre, pour le modèle $G_{\mathrm{a}}$, les deux contributions du bruit propre et cisaillement sont complémentaires l'une de l'autre. Pour les angles $\theta>45^{\circ}$ (respectivement $\theta<45^{\circ}$ ), c'est l'intensité du bruit propre (respectivement du bruit de cisaillement) qui domine de telle manière que l'intensité totale modélise correctement les données de Lush (cf. Fig. 5). Cependant pour la vitesse de $125 \mathrm{~m} / \mathrm{s}$, ce modèle sous-estime l'intensité mesurée pour les petits angles. En fait, il est bon de rappeler que nous avons pris une valeur fixe pour le rapport $\Delta=1 / 3$, et que le facteur $N$ est pratiquement nul. Or on sait bien que si la vitesse de sortie diminue, les structures turbulentes ont tendance à devenir plus isotropes et le rapport $\Delta$ tend alors vers 1 . Ce retour vers l'isotropie est caractérisé par une directivité qui sera proche de celle du modèle $R_{\mathrm{a}}$, et on aura ainsi une intensité acoustique aux petits angles plus élevée.

4.5 RÉPARTITION SPATIO-FRÉQUENTIELLE DE LA PUISSANCE ACOUSTIQUE. — L'expression du spectre de la puissance acoustique par unité de volume de jet peut être écrite à partir de l'expression du spectre de l'intensité acoustique directionnelle $I_{\omega}(x, \theta \mid y)$ en intégrant sur toutes les directions angulaires $\theta$ (voir [1] pour les expressions analytiques de $I_{\omega}(x, \theta \mid \mathbf{y})$ ) :

$$
W_{\omega}(\mid \mathbf{y})=2 \pi x^{2} \int_{\theta_{\min }}^{\theta_{\max }} I_{\omega}(x, \theta \mid \mathbf{y}) \sin \theta \mathrm{d} \theta
$$

Pour obtenir une représentation plus synthétique de l'évolution du spectre de la puissance acoustique en fonction du nombre de Strouhal St $\left(\mathrm{St}=\omega D /\left(2 \pi U_{1 \mathrm{~b}}\right)\right.$ ), et de la distance axiale $y_{1}$, nous avons intégré la relation (31) sur chaque section droite du jet pour obtenir le spectre de 


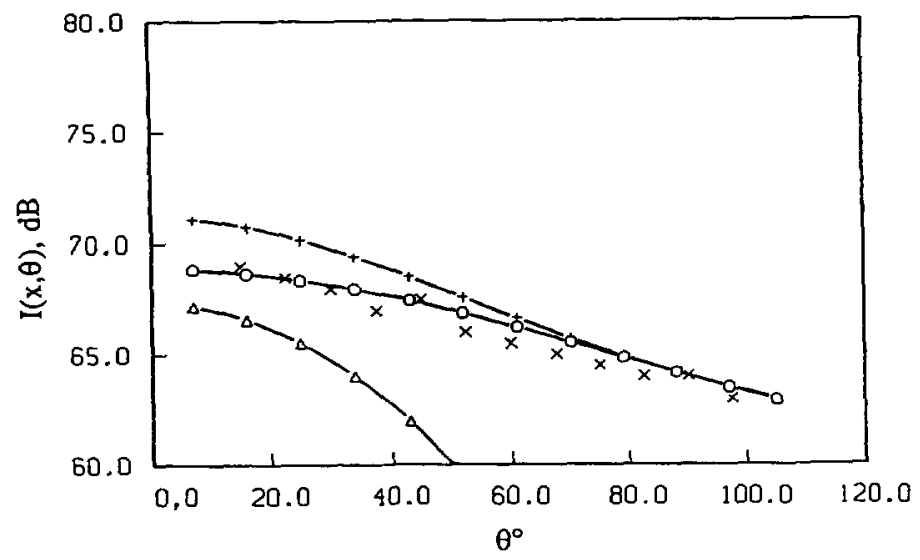

a)

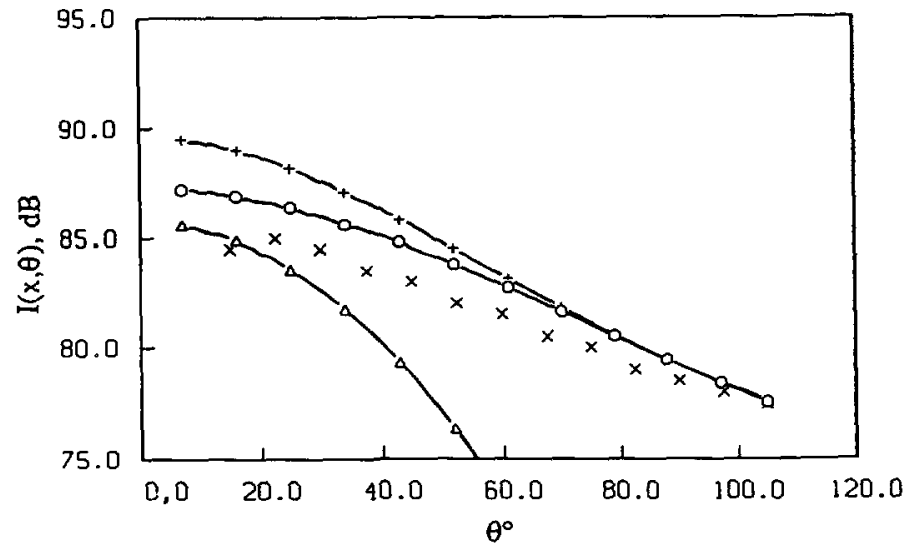

b)

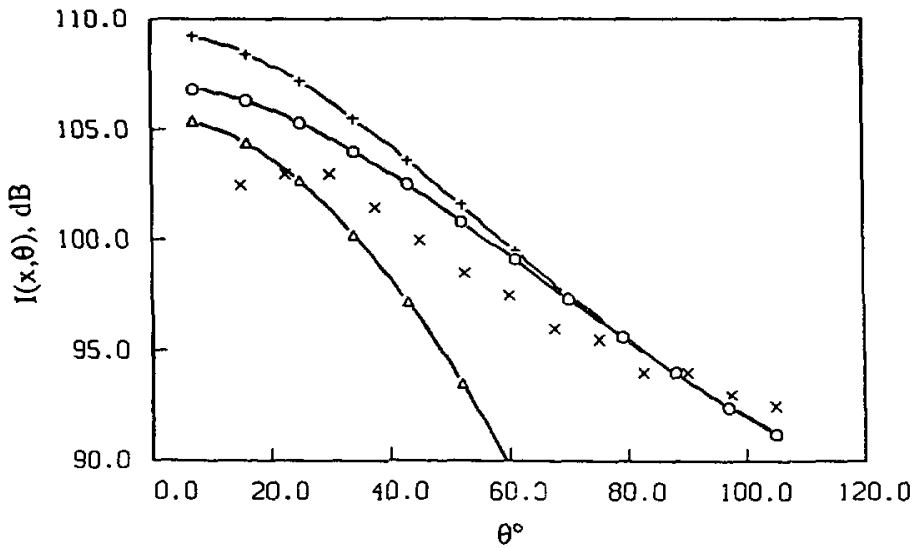

c)

Fig. 4. - Variation de l'intensité acoustique directionnelle du bruit propre et du bruit de cisaillement en fonction de l'angle d'observation $\theta$ pour le modèle $R_{\mathrm{a}}$. a) $125 \mathrm{~m} / \mathrm{s}$, b) $195 \mathrm{~m} / \mathrm{s}$, c) $300 \mathrm{~m} / \mathrm{s}$. ((O) B. Propre, $(\Delta)$ B. Cisaillement, $(+)$ B. Total $(x)$ Lush).

[Variation of the acoustic directional intensity of self noise and shear noise as a function of the angle of observation $\theta$ for the $R_{\mathrm{a}}$ model. a) $125 \mathrm{~m} / \mathrm{s}$, b) $195 \mathrm{~m} / \mathrm{s}$, c) $300 \mathrm{~m} / \mathrm{s}$. ((O) Self N., ( $\triangle$ ) Shear N., (+) Total N., $(x)$ Lush).] 


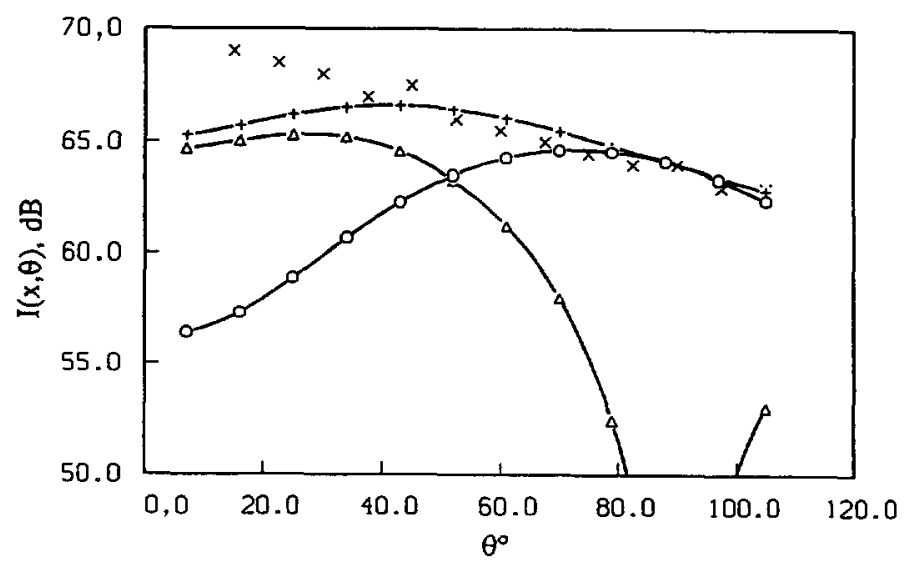

a)

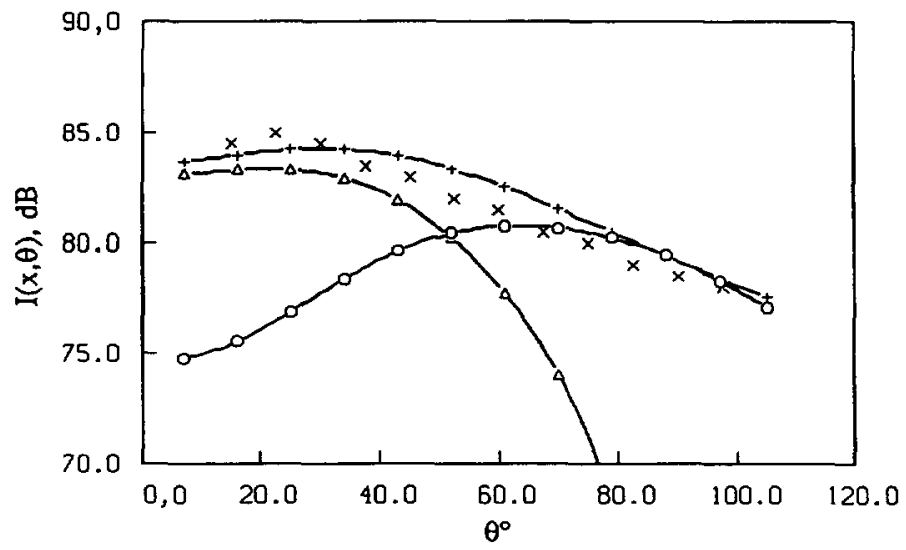

b)

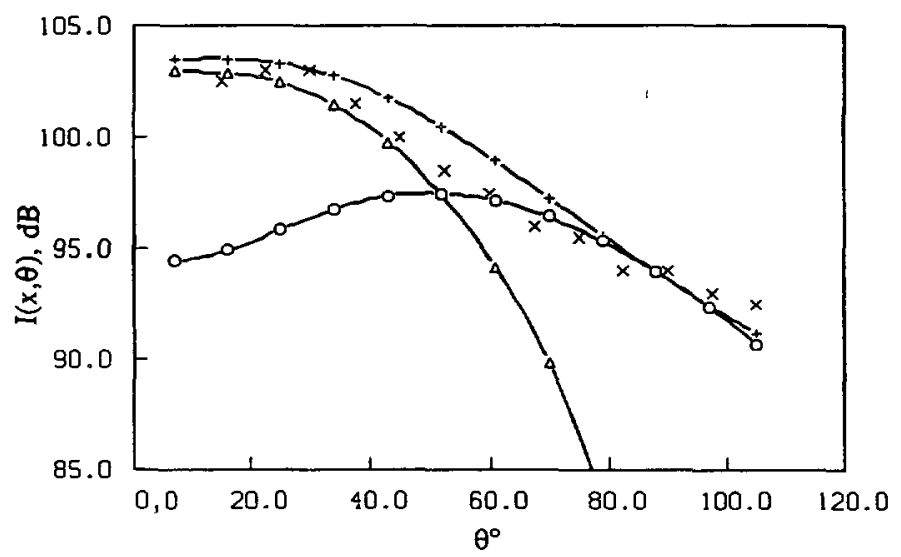

c)

Fig. 5. - Variation de l'intensité acoustique directionnelle du bruit propre et du bruit de cisaillement en fonction de l'angle d'observation $\theta$ pour le modèle $G_{\mathrm{a}}$. a) $125 \mathrm{~m} / \mathrm{s}$, b) $195 \mathrm{~m} / \mathrm{s}$, c) $300 \mathrm{~m} / \mathrm{s}$. ((O) B. Propre, $(\Delta)$ B. Cisaillement, $(+)$ B. Total, $(x)$ Lush).

[Variation of the acoustic directional intensity of self noise and shear noise as a function of the angle of observation $\theta$ for the $G_{\mathrm{a}}$ model. a) $125 \mathrm{~m} / \mathrm{s}$, b) $195 \mathrm{~m} / \mathrm{s}$, c) $300 \mathrm{~m} / \mathrm{s}$. ((O) Self N., ( $\left.\Delta\right)$ Shear N., (+) Total N., ( $x$ ) Lush).] 
la puissance acoustique par unité de longueur $\mathrm{d} W_{\omega} / \mathrm{d} y_{1}$.

$$
\frac{\mathrm{d} W_{\omega}}{\mathrm{d} y_{1}}=\int_{\left(y_{2} \text { et } y_{3}\right)} W_{\omega}(\mid \mathbf{y}) \mathrm{d} y_{2} \mathrm{~d} y_{3} .
$$

La figure 6 montre qu'à faible distance du plan d'éjection, le maximum de $\mathrm{d} W_{\omega} / \mathrm{d} y_{1}$ se situe aux hautes fréquences $(\mathrm{St}=3$ ), puis ce maximum se déplace vers des fréquences intermédiaires $(\mathrm{St}=0,7-2)$ à plus grande distance de la buse d'éjection. A partir de $16 \mathrm{D}$, la quantité $\mathrm{d} W_{\omega} / \mathrm{d} y_{1}$ est centrée sur les fréquences basses $(\mathrm{St}<0,5$ ). En effet, les tourbillons à fréquence élevée formés à proximité de la tuyère sont responsables de l'émission acoustique à haute fréquence, et au fur et à mesure qu'on s'éloigne de la tuyère, les échelles des tourbillons augmentent et leurs temps caractéristiques s'allongent et l'émission se fait par conséquent à des fréquences de plus en plus basses.

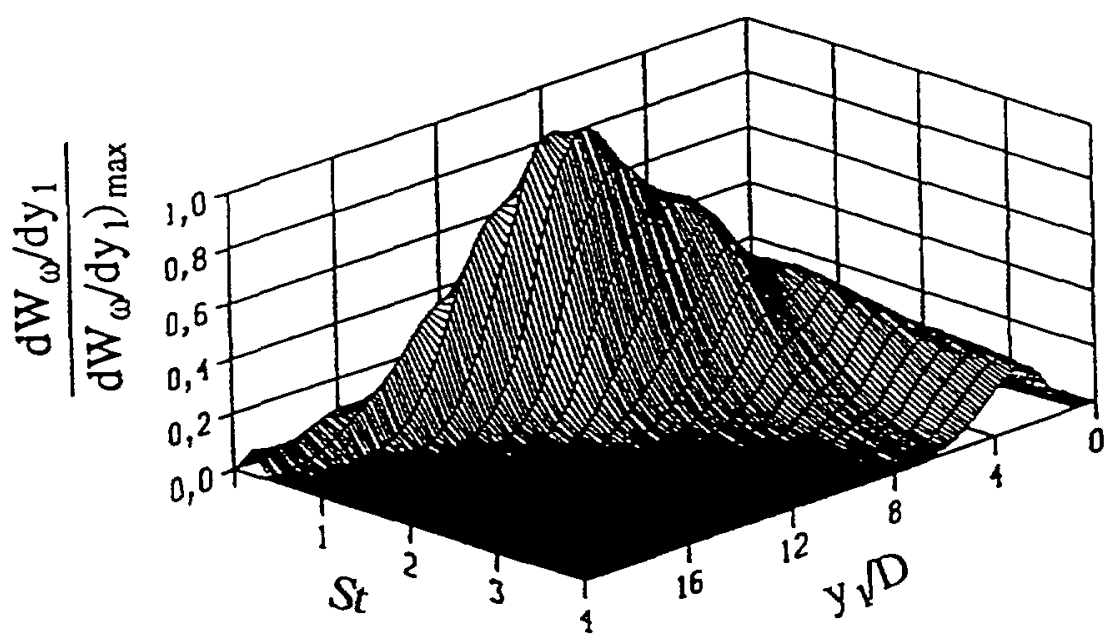

Fig. 6. - Variation du spectre de la puissance acoustique par unité de longueur en fonction du nombre de Stouhal St et $y_{1} / D$ (modèle $G_{\mathrm{a}}$ pour $300 \mathrm{~m} / \mathrm{s}$ ).

[Variation of the acoustic power spectrum per unit length as a function of the Stouhal number St and $y_{1} / D\left(G_{\mathrm{a}}\right.$ model for $\left.300 \mathrm{~m} / \mathrm{s}\right)$.]

\section{Détermination de l'émission acoustique pour des jets coaxiaux.}

5.1 Position du PROBLÈme. - On se propose d'appliquer le modèle de Goldstein adapté $G_{\text {a }}$ pour étudier la diminution de l'émission acoustique pour des jets coaxiaux. Cette application consiste à simuler les expériences réalisées par Juvé et al. [11] sur deux jets coaxiaux subsoniques à température ambiante. Ces expériences consistaient à déterminer le minimum de l'émission acoustique en faisant varier la vitesse du jet secondaire (ou annulaire extérieur). Notre objectif sera, pour une configuration géométrique donnée, de retrouver la vitesse du jet secondaire correspondant à ce minimum d'émission acoustique et de prévoir la diminution de l'intensité acoustique mesurée pour cette vitesse. La configuration choisie est celle qui correspond à un jet primaire de diamètre $D_{\mathrm{p}}=30 \mathrm{~mm}$ et de vitesse de sortie $U_{1 \mathrm{p}}=130 \mathrm{~m} / \mathrm{s}$, et à un jet secondaire de diamètre $D_{\mathrm{s}}=100 \mathrm{~mm}$ et de vitesse de sortie $U_{1 \mathrm{~s}}=0,26,52,78$ et $91 \mathrm{~m} / \mathrm{s}$. La figure 7 donne une représentation schématique de la 


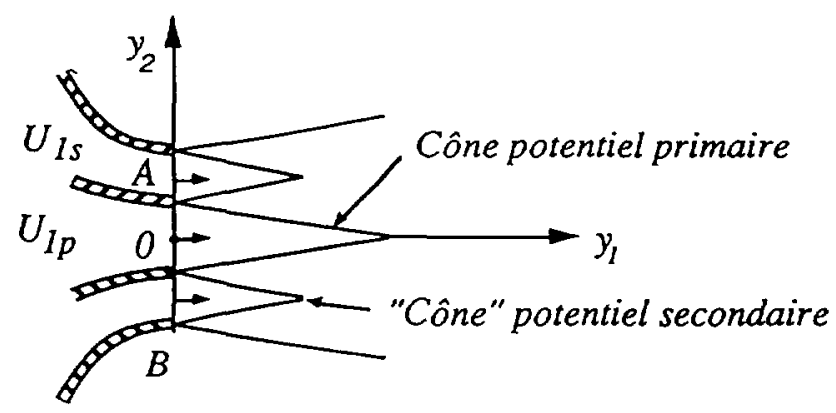

Fig. 7. - Configuration des deux jets coaxiaux. ( $U_{1 \mathrm{p}}$ et $U_{1 \mathrm{~s}}$ vitesses du jet primaire et secondaire à la buse, $D_{\mathrm{p}}$ et $D_{\mathrm{s}}$ diamètres des buses intérieure et extérieure : $2 \mathrm{OA}=D_{\mathrm{p}}, 2 \mathrm{OB}=D_{\mathrm{s}}$ ).

[Coaxial jet configuration. ( $U_{1 \mathrm{p}}$ and $U_{1 \mathrm{~s}}$ velocities of primary and secondary jets at the nozzle, $D_{\mathrm{p}}$ and $D_{\mathrm{s}}$ diameters of the inner and outer nozzles : $2 \mathrm{OA}=D_{\mathrm{p}}, 2 \mathrm{OB}=D_{\mathrm{s}}$ ).

configuration étudiée. La démarche adoptée pour calculer l'émission acoustique est basée tout d'abord sur un calcul numérique de l'écoulement moyen et des grandeurs statistiques par le code ULYSSE. Puis à l'aide du modèle $G_{\mathrm{a}}$, défini plus haut, on remonte aux grandeurs acoustiques désirées et enfin on compare ces résultats aux mesures expérimentales de Juvé $e t$ al. [11].

En comparant nos calculs des grandeurs caractérisant l'écoulement sur le plan aérodynamique (profils de la vitesse axiale moyenne $U_{1}$, de l'énergie cinétique turbulente axiale

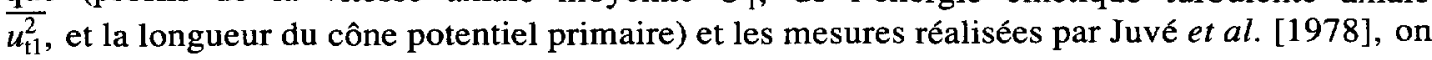
trouve un bon accord pour les trois rapports de vitesse $\lambda=U_{1 \mathrm{~s}} / U_{1 \mathrm{p}}=0,0,4$ et 0,7 [1].

5.2 VAlidation du CAlCUl DE L'INTENSITÉ ACOUSTIQUe DiRECTIONNELle. - Juvé et al. [11] ont mesuré l'évolution de l'intensité acoustique directionnelle $I(x, \theta)$ due à la totalité du jet avec le rapport $\lambda$. Ces mesures sont effectuées en champ lointain, à une distance de $2,5 \mathrm{~m}$ de l'origine des jets et pour un angle $\theta=90^{\circ}$. Pour cet angle particulier, la connaissance du profil exact de la vitesse de convection $U_{1 \mathrm{c}}$ n'est plus nécessaire car l'amplification due au facteur de convection $C_{\mathrm{m}}$ défini par la relation (20) devient négligeable. En effet, le tableau I montre que le facteur de calage $F_{\mathrm{C}}^{\mathrm{G}}$ pour le modèle de Goldstein reste pratiquement le même dans toutes les configurations envisagées pour la modélisation de $C_{\mathrm{m}}$.

Le calcul numérique de $I(x, \theta)$ a été réalisé pour les cinq rapports de vitesse. Sur la figure 8 , on voit que le modèle $G_{\mathrm{a}}$ reproduit correctement le rapport $\lambda=0,4$ pour lequel l'intensité acoustique est minimale. Cependant l'atténuation prévue par le code par rapport au jet simple est de $6,9 \mathrm{~dB}$, alors que celle mesurée expérimentalement est de 7,9 dB. L'écart de 1,3 dB observé pour $\lambda=0$ peut être justifié par le fait qu'il existe un décalage entre les mesures effectuées par Juvé et al. [11] et Lush [17], pour une même configuration. Pour le jet simple de diamètre $D_{\mathrm{L}}=25 \mathrm{~mm}$ et à $125 \mathrm{~m} / \mathrm{s}$, la valeur de $I_{\mathrm{L}}\left(x=3 \mathrm{~m}, \theta=90^{\circ}\right)$ de Lush est de $64,0 \mathrm{~dB}$. Pour comparer $I_{\mathrm{L}}$ à la valeur de Juvé $I_{\mathrm{J}}\left(x=2,5 \mathrm{~m}, \theta=90^{\circ}\right)=69,4 \mathrm{~dB}$ pour le jet de diamètre $D_{\mathrm{J}}=30 \mathrm{~mm}$ et une vitesse de $130 \mathrm{~m} / \mathrm{s}$, il faut corriger la valeur de Lush à l'aide de la loi dimensionnelle en $U^{8} D^{2} / x^{2}$. On trouve ainsi une intensité corrigée :

$$
I_{\mathrm{L}}^{\mathrm{c}}=I_{\mathrm{L}}+10 \log _{10}\left(\frac{130}{125}\right)^{8}+10 \log _{10}\left(\frac{30}{25}\right)^{2}-10 \log _{10}\left(\frac{2,5}{3,0}\right)^{2}=68,5 \mathrm{~dB}
$$

et l'écart $I_{\mathrm{J}}-I_{\mathrm{L}}^{\mathrm{c}}=0,9 \mathrm{~dB}$. Comme le facteur de calage $F_{\mathrm{C}}^{\mathrm{G}}$ du modèle $G_{\mathrm{a}}$ est calculé à partir 


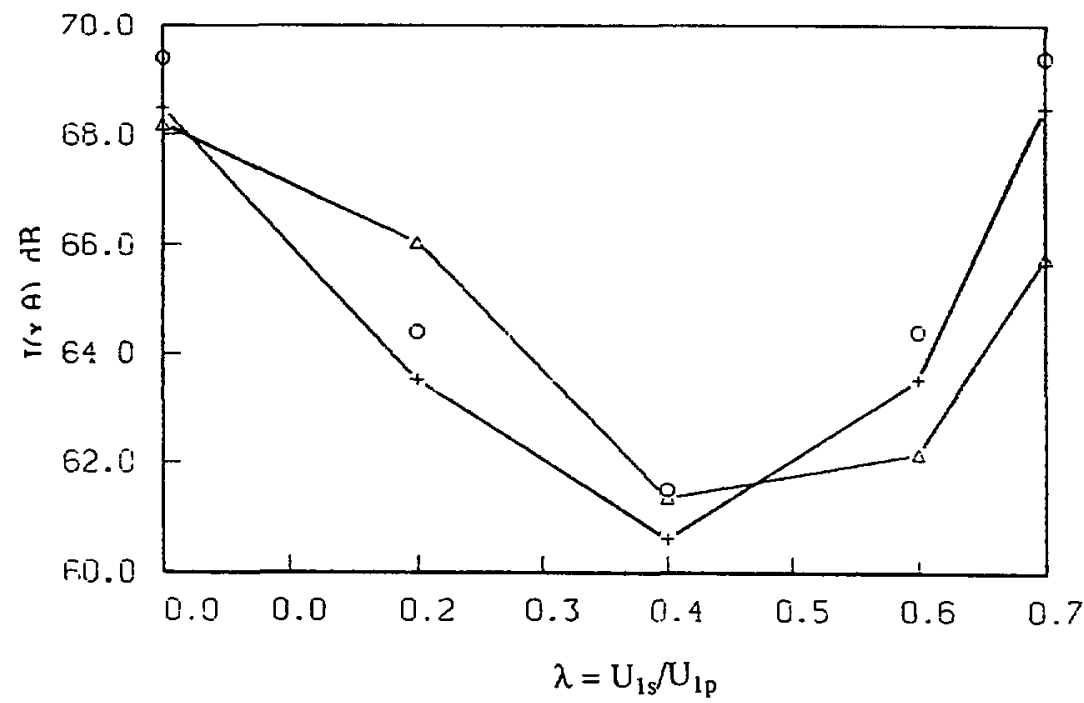

Fig. 8. - Comparaison entre l'intensité acoustique directionnelle calculée numériquement (Modèle $G_{\mathrm{a}}$ ) et les valeurs expérimentales de Juvé et al. [11] pour $\theta=90^{\circ}$ et $x=2,5 \mathrm{~m}$. ((O) Juvé, $(\Delta)$ modèle $G_{\mathrm{a}},(+)$ Juvé corrigé).

[Comparison between numerical acoustic directional intensity $\left(G_{\mathrm{a}}\right.$ model) and experimental values of Juvé et al. [11] for $\theta=90^{\circ}$ and $x=2.5 \mathrm{~m}$. ((O) Juvé, ( $\left.\Delta\right) G_{\mathrm{a}}$ model, (+) Juvé corrected).]

des données de Lush, il faut donc diminuer les valeurs expérimentales de Juvé de la figure 8 de $0,9 \mathrm{~dB}$ pour faire une comparaison justifiée avec le modèle $G_{\mathrm{a}}$. Les écarts plus importants observés pour $\lambda=0,6$ et 0,7 sont dus au fait que la totalité des sources acoustiques, pour ces cas, n'est pas contenue dans le domaine de calcul considéré. En effet, ce domaine s'étend jusqu'à $30 D_{\mathrm{p}}$ mais cette longueur reste insuffisante pour les grandes vitesses du jet secondaire.

\section{Conclusion.}

Nous montrons dans cette étude qu'il est possible de prévoir le bruit généré par des jets turbulents libres à partir des grandeurs locales de l'écoulement moyen et des grandeurs statistiques de la turbulence. En utilisant un code numérique de turbulence de type $K-\varepsilon$ et à l'aide d'un modèle théorique $G_{\mathrm{a}}$ (modèle de Goldstein adapté), nous avons réussi à retrouver correctement l'évolution de la puissance acoustique $W$ en fonction de la vitesse de sortie, ainsi que la variation de l'intensité acoustique directionnelle en fonction de l'angle d'observation. Ce modèle développé dans le cadre d'une turbulence axisymétrique superposée à un écoulement moyen est mieux adapté aux écoulements cisaillés que le modèle modifié de Ribner (modèle $R_{\mathrm{a}}$ ) associé à une turbulence isotrope. De même, une comparaison entre les différents modèles basés sur la théorie de Lighthill a montré que le modèle $G_{\mathrm{a}}$ donne la meilleure directivité de l'intensité acoustique aux vitesses élevées. D'autre part, avec le modèle $G_{\mathrm{a}}$, nous avons étudié les répartitions spatiales et fréquentielles des sources acoustiques. Ainsi nous avons montré en particulier que les sources acoustiques dans la zone de mélange sont à fréquence élevée alors que celles de la zone développée sont à fréquence plus basse.

Le modèle $G_{\mathrm{a}}$ peut être utilisé comme un outil d'optimisation permettant une prévision des évolutions des grandeurs acoustiques en fonction des paramètres d'étude et ceci pour des 
configurations variées de jets libres. En effet, son utilisation pour le calcul du bruit émis par deux jets coaxiaux a montré qu'il était possible de retrouver le minimum de l'émission acoustique obtenu en faisant varier la vitesse du jet secondaire et la diminution de l'intensité acoustique a été estimée à $1 \mathrm{~dB}$ près. Toutefois, il faut noter que la qualité des prévisions acoustiques déduites du modèle $G_{\mathrm{a}}$ dépend de façon significative du calcul aérodynamique. En ce sens, une amélioration des prévisions sera possible en utilisant des modèles de turbulence plus évolués que le modèle $K-\varepsilon$. On pourra citer, par exemple, les modèles de second ordre de type $R_{\imath j}-\varepsilon$, ou bien les modèles algébriques des corrélations, moins sophistiqués, qui permettent en principe de mieux prévoir les évolutions des différents termes $\overline{u_{t i} u_{t}}$ du tenseur de Reynolds. Avec ces types de modèles, on pourrait représenter plus correctement les rapports $N$ et $\Delta$ du modèle $G_{\mathrm{a}}$.

Pour terminer il faut signaler que le modèle de bruit développé ici souffre de deux limitations. La première est l'inaptitude du modèle à tenir compte du phénomène de réfraction des ondes acoustiques par l'écoulement moyen. Ceci se traduit par une atténuation prononcée sur l'intensité acoustique directionnelle pour des angles $\theta<15^{\circ}$. La deuxième est liée au fait que la modélisation est en principe uniquement adaptée au cas des jets libres, en l'absence de parois.

\section{Bibliographie}

[1] Bechara W., Modélisation du bruit d'écoulements turbulents libres, Thèse de Doctorat en Mécanique des Fluides et Acoustique, Ecole Centrale paris (1992) 1992-02.

[2] Berman C. and Ramos J., Simultaneous computations of jet turbulence and noise, AIAA89-1091, 3rd AIAA Aeroacoustics Conference (San-Antonio, TX, 1989) pp. 1-11.

[3] BILANIN A. J. and HIRSH J. E., Application of second order turbulent modeling to the prediction of radiated aerodynamic sound, NASA (1978) 2994.

[4] Davies P. O. A. L., Fisher M. J. and BarRatT M. J., The characteristics of the turbulence in the mixing region of a round jet, J. Fluid Mech. 15 (1963) 337-367.

[5] Ffowcs Williams J. E., The noise from turbulence convected at high speed, Philos. Trans. R. Soc. A 225 (1963) 469-503.

[6] Goldstein M. E. and Rosenbaum B., Effect of anisotropic turbulence on aerodynamic noise, $J$. Acoust. Soc. Am. 54 (1973) 630-645.

[7] Goldstein M. E., Aeroacoustics (McGraw-Hill International Book Compagny, 1976).

[8] Grant M. L., The large eddies of turbulent motion, J. Fluid Mech. 4 (1958) 149.

[8a] Ha Minh H., Physique et modélisation de la turbulence en écoulement de fluides, Ecole de printemps de mécanique des fluides numériques (Aussois, 1991).

[9] Hecht A. M., Teske M. E. and BIlanin A. J., Predicting aerodynamic sound utilizing a two-point, two-time turbulence theory, AIAA81-2023, AIAA 7th Aeroacoustics Conference (Palta Alto, California, 1981) pp. 1-20.

[10] Jones I. S. F., Aerodynamic noise dependent mean shear, J. Fluid Mech. 33 (1968) 65-72.

[10a] JONES W. P., LAUNDER B. E., The prediction of Laminarization with a two-equation Model of Turbulence, Int. J. Heat Mass Trans. 15 (1972) 301-314.

[11] Juve D., Batallle J. and Comte-Bellot G., Bruit de jets coaxiaux froids subsoniques, J. Mech. Appl. 2 (1978) 385-398.

[12] KRISHNAPPA G., In aerodynamic noise, Proceedings of the symposium (Toronto, Canada, 1968) pp. 89-110.

[13] LAURENCE D., Code ULYSSE : Note de principe, EDF-DER, (1989) HE-41/89-32B.

[14] LighthiLl M. J., On sound generated aerodynamically. Part I, Proc. R. Soc. London I (1952) 564587. 
[15] Lighthill M. J., On sound generated aerodynamically. Part II, Proc. R. Soc. London II (1954) 132.

[16] LilLEY G. M., On the noise from jets, (1958) ARC 20, 376-N40-FM 2724.

[17] Lush P. A., Measurements of subsonic jet noise and comparaison with theory, J. Fluid Mech. 71 (1971) 319-332.

[18] PAo S. P. and Lowson M. V., Some applications of jet noise theory, AIAA70-233, AIAA 8th Aerospace Sciences Meeting Conference (New York, 1970).

[19] Proudman I., The generation of sound by isotropic turbulence, Proc. R. Soc. London (1952) 119132.

[20] RibNeR H. S., On spectra and directivity of jet noise, J. Acoust. Soc. Am. 35 (1963) 614-616.

[21] RiBner H. S., Advances in applied mechanics (Academic Press, New York, 1964) pp. 103-182.

[22] RIBNER H. S., Quadrupole correlations governing the pattern of jet noise, J. Fluid Mech. 38 (1969) $1-24$.

[23] RIBNER H. S., On the role of shear term in jet noise, J. Sound Vib. 52 (1977) 121-132. 